Published in "Neuroscience 357(): 303-324, 2017"

which should be cited to refer to this work.

\title{
ROLE OF PRIMARY MOTOR CORTEX IN THE CONTROL OF MANUAL DEXTERITY ASSESSED VIA SEQUENTIAL BILATERAL LESION IN THE ADULT MACAQUE MONKEY: A CASE STUDY
}

\section{JULIE SAVIDAN, MÉLANIE KAESER, ABDERRAOUF BELHAJ-SAIIF, ERIC SCHMIDLIN AND ERIC M. ROUILLER *}

Department of Medicine, Fribourg Centre for Cognition, University of Fribourg, Chemin du Musée 5, CH-1700 Fribourg, Switzerland

\begin{abstract}
From a case study, we describe the impact of unilateral lesion of the hand area in the primary motor cortex (M1) on manual dexterity and the role of the intact contralesional M1 in long-term functional recovery. An adult macaque monkey performed two manual dexterity tasks: (i) "modified Brinkman board" task, assessed simple precision grip versus complex precision grip, the latter involved a hand postural adjustment; (ii) "modified Klüver board" task, assessed movements ranging from power grip to precision grip, pre-shaping and grasping. Two consecutive unilateral M1 lesions targeted the hand area of each hemisphere, the second lesion was performed after stable, though incomplete, functional recovery from the primary lesion. Following each lesion, the manual dexterity of the contralesional hand was affected in a comparable manner, effects being progressively more deleterious from power grip to simple and then complex precision grips. Both tasks yielded consistent data, namely that the secondary M1 lesion did not have a significant impact on the recovered performance from the primary M1 lesion, which took place 5 months earlier. In conclusion, the intact contralesional M1 did not play a major role in the long-term functional recovery from a primary M1 lesion targeted to the hand area. (C) 2017 IBRO. Published by Elsevier Ltd. All rights reserved.
\end{abstract}

Key words: non-human primate, motor control, functional $r$ ecovery, cortical lesion, reaching, grasping.

\section{INTRODUCTION}

Strokes affecting the primary motor cortex (M1) hand area have been reported to induce a wide range of deficits on various aspects of reach and grasp movements, depending on the size and precise location of the injury,

\footnotetext{
${ }^{*}$ Corresponding author.

E-mail addresses: julie.savidan@unifr.ch (J. Savidan), melanie. kaeser@unifr.ch (M. Kaeser), abderraouf.belhaj@unifr.ch (A. Belhaj-Saïf), eric.schmidlin@unifr.ch (E. Schmidlin), eric.rouiller@unifr. ch (E. M. Rouiller)

Abbreviations: Ac-P, acute period post-lesion; CT, contact time; ICMS intracortical microstimulation; M1, primary motor cortex; PreL, prelesion period; Rec-P, recovery period; PI-P, plateau period.
}

which have been reproduced to some extent by transient inactivation (e.g. Rizzolatti and Luppino, 2001; Shelton and Reding, 2001; Olivier et al., 2007; Brown and Teskey, 2014). In monkeys, alterations of hand movements have been reported after unilateral transient inactivation or permanent lesion of $\mathrm{M} 1$ followed by the occurrence of compensatory strategies, affecting motor parameters such as force (Brochier et al., 1999), trajectory (Cirstea and Levin, 2000), precision grip (Brochier et al., 1999; Darling et al., 2009, 2011b, 2013, 2014; Kermadi et al., 1997; Liu and Rouiller, 1999; Kaeser et al., 2010; Hoogewoud et al., 2013; Morecraft et al., 2015, 2016; Wyss et al., 2013; Murata et al., 2015), flexion-extension (Schieber and Poliakov, 1998) and wrist movement (Hoffman and Strick, 1995). Depending on the size and location of the injury, spontaneous recovery occurs to a variable extent, though it is generally incomplete. The precise mechanisms and anatomical basis for spontaneous motor recovery remain unclear and variable (for review: Nudo, 2006; Nudo and Barbay, 2014). There is evidence that the perilesional M1 and/or the ipsilesional ventral premotor cortex (PMv) play a role in the functional recovery (e.g. Glees and Cole, 1950; Liu and Rouiller, 1999; Dancause et al., 2005; Wyss et al., 2013; Murata et al., 2015). In contrast, the role of the contralesional intact M1 remains controversial. The intact M1 exhibits a high level of activity following a lesion or stroke (Babiloni et al., 1999; Gonzalez et al., 2004), although the role of this increased activity in spontaneous recovery remains a matter of debate and appears to be restricted to the early periods of recovery (Salmelin et al., 1995; Netz et al., 1997; Rehme et al., 2011). In macaque monkeys (Liu and Rouiller, 1999), the spontaneous functional recovery following a small $\mathrm{M} 1$ lesion was not affected by subsequent transient inactivation of the contralateral intact $\mathrm{M} 1$, in contrast to the rat model in which such inactivation abolished the recovery following large unilateral stroke (Biernaskie et al., 2005). These data suggest that the size of the unilateral M1 lesion may trigger different mechanisms of recovery, involving or not the intact M1. As reported recently (Morecraft et al., 2016), the size of the lesion in the frontal lobe as well as the spread of the lesion in the parietal lobe determined the degree of impact of the intact contralesional M1 in the functional recovery in rhesus monkeys. Nevertheless, the notion that the intact contralesional M1 provides systematic, full, and direct support for the spontaneous functional recovery occurring following a stroke appears to be controversial, albeit there 
is evidence supporting an additional contribution of the intact M1 to that of ipsilesional non-primary motor areas (Jaillard et al., 2005; Dancause, 2006; Dancause et al., 2015). Furthermore, the role of the contralesional intact M1 may be restricted to specific periods of the functional recovery (e.g. acute period and not plateau period). It has been suggested that bilateral manual control exerted by M1 depends on both the task complexity and the size of the cortical lesion (Shibasaki et al., 1993; Salmelin et al., 1995; Chen et al., 1997; Biernaskie et al., 2005; Bashir et al., 2012). Consequently, depending on the motor task, the role of the intact M1 in functional recovery may be either enhanced or masked.

Across laboratories, two motor tasks have been extensively used to assess different aspects of hand grasp movements in non-human primates: $(i)$ the modified Brinkman board task testing precision grip in different hand positions (e.g. Bashir et al., 2012; Freund et al., 2006, 2009; Kaeser et al., 2010, 2011, 2013, 2014; Liu and Rouiller, 1999; Schmidlin et al., 2004, 2005, 2011; Wyss et al., 2013), derived from an early initial version (Brinkman and Kuypers, 1973; Brinkman, 1984); (ii) the modified Klüver board task to assess various types of finger grip, ranging from power grip to precision grip (e.g. Nudo et al., 1992; Xerri et al., 1998; Murata et al., 2008; Milliken et al., 2013; Qi et al., 2013; Sugiyama et al., 2013).

The present case report in a non-human primate aims at investigating the role of the contralesional, intact M1 on the incomplete functional recovery of manual dexterity following a unilateral M1 lesion affecting the hand area. To this aim, following a plateau of functional recovery, the contralesional intact $M 1$ was subjected to a permanent lesion. Earlier studies have reported in a qualitative manner the effects of such sequential permanent lesion of the motor cortex, either in chimpanzees (Brown and Sherrington, 1913; Leyton and Sherrington, 1917) or in macaques (Ogden and Franz, 1917; Kennard, 1942). Overall, these early studies support the notion that the intact motor cortex does not play a major role in the recovery from the primary lesion. However, interpretation of these early results is limited by the qualitative nature of the reported data, which were largely based on observations of the animals' daily activities in their housing area. Indeed, the fact that the intact motor cortex is involved in the control of complex voluntary movement (e.g. manual dexterity) suggests that such observational assessment may not be adequate to detect the full impact of a lesion. The present study goes beyond qualitative measures by introducing several quantitative assessments of precise and complementary motor parameters, across sufficiently long time windows before and after the sequential lesions, for both hands. Two manual motor tests, namely the modified Brinkman board task and the modified Klüver board task, representing tasks of various complexities (e.g. type of grip, different posture), were used in parallel to assess various attributes of manual dexterity. More specifically, we tested the hypotheses that: (1) after unilateral M1 lesion restricted to the hand area, several aspects of fine manual dexterity are differentially affected; (2) after a unilateral
M1 lesion restricted to the hand area, a secondary permanent lesion of the intact M1 does not have an impact on the previous spontaneous functional recovery of the hand affected by the primary M1 lesion.

\section{EXPERIMENTAL PROCEDURES}

\section{General survey of the experiment}

The present case study was conducted on one adult male monkey (Macaca fascicularis; $9 \mathrm{~kg}$ ), 10 years old at the time of euthanasia (Mk-DG). All experiments were carried out in accordance to the Guide for Care and Use of Laboratory Animals (ISBN 0-309-05377-3; 1996) and approved by local veterinary authorities (authorizations No 192/07, 19017 and 22010), including the ethical assessment by the local (cantonal) Survey Committee on Animal Experimentation and a final acceptance delivered by the Federal Veterinary Office (BVET, Bern, Switzerland). The monkey was purchased from a certified supplier (Harlan Buckshire, USA; monkey bred in China, followed by quarantine in the European Harlan Center, Milano, Italy). The housing conditions in groups of 2-5 monkeys can be seen on-line: www.unifr.ch/ spccr/about/housing.

Fig. 1 summarizes the time course of the present case report. The animal was first trained to perform two behavioral tasks with each hand: the "modified Brinkman board" and the "modified Klüver board" tasks. Following a "learning" period in order to reach a stable performance (see Chatagny et al., 2013; Kaeser et al., 2014), 3 months of pre-lesion behavioral data were collected. During this pre-lesion period (PreL), a structural MRI of reference was acquired (method described in Peuser et al., 2011); in addition, a chronic chamber and epidural grid were implanted over the M1 hand area on the left hemisphere. Once the monkey recovered from the surgery, the behavioral tasks resumed. Additionally intracortical microstimulation (ICMS) sessions were performed twice a week alternating days with the behavioral tasks, to map the targeted hand area in the left M1. Both the MRI and the ICMS map were used to design a focal and permanent primary lesion in the hand area of M1, performed by infusion of ibotenic acid. Functional recovery was assessed over five months, based on the two behavioral tasks and during this period the chronic chamber was removed. The time window following the primary left $M 1$ lesion was comprised of three periods specifically defined for the different behavioral parameters of the contralesional forelimb (Fig. 1): (1) The acute period post-lesion 1 (Ac-P1; characterized by a behavioral score of zero). (2) The recovery period (Rec-P1; characterized by a progressive increase of score). (3) The plateau period of recovery ( $\mathrm{PI}-\mathrm{P} 1$; characterized by a stable recovered score). A second MRI scan was then acquired to visualize the primary lesion. Four months after the primary M1 lesion, a second chronic chamber was implanted over the contralesional intact M1 hand area, on the right hemisphere. Once the monkey recovered from the surgery, the daily behavioral sessions resumed and were again alternated with ICMS sessions in order to map the hand region 
Behavioral time line (consecutive periods)

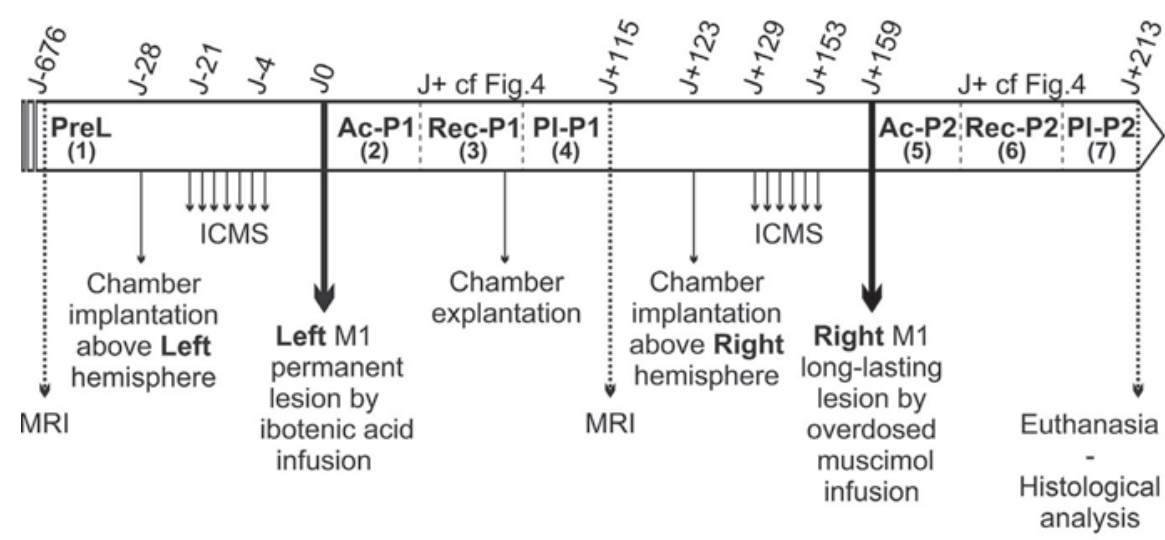

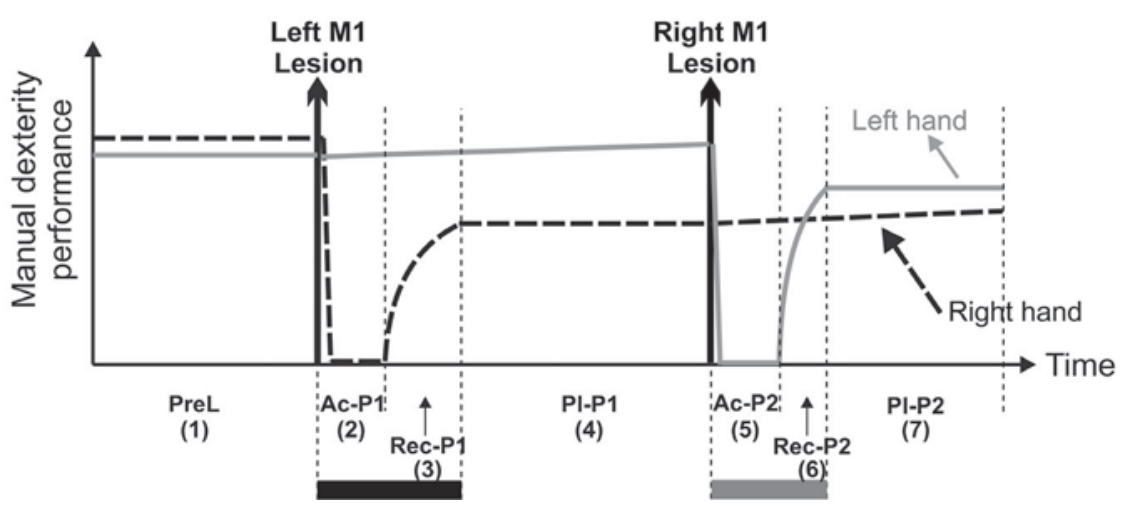

Fig. 1. General survey. Top panel: Schematic representation of the experimental protocol (time line) in monkey Mk-DG, showing the succession of the different interventions, in parallel to the continuous behavioral assessment. The days corresponding to the main events are indicated on top of the time line, with respect to the day of the primary M1 lesion J0: negative days (e.g. J-28) are for pre-primary lesion dates and positive days (e.g. J + 115) are for days post-primary lesion. The different periods represent the time-intervals of the functional recovery based on the performance of the affected hand for each behavioral parameters (the numbers in bracket are used to refer the corresponding time periods in the results graphs Figs. 5-7): PreL (1) for the PreLesion period, Ac-P1 (2) for the Acute Period during which the lesioned hand was completely unable to perform the task following the primary lesion in the left M1 hand area, Rec-P1 (3) for the subsequent Period of Recovery, PI-P1 (4) when the animal reached a stable recovered performance, referred to as Plateau Period. The Ac-P2 (5), Rec-P2 (6) and PI-P2 (7) labels characterize equivalent periods following the secondary lesion in the right $\mathrm{M} 1$ hand area. The precise dates in the time windows Ac-P1, Rec-P1, PI-P1, Ac-P2, Rec-P2 and PI-P2 for each of the parameters are indicated in Fig. 4. Bottom panel: The same periods in the functional recovery process are shown superimposed onto an illustration of a typical manual dexterity performance curve expected in case of a primary unilateral M1 lesion on the left hemisphere, followed by a secondary unilateral $\mathrm{M} 1$ lesion on the right hemisphere. The motor performance is schematized as a function of time by the black dashed curve for the right hand and the gray solid curve for the left hand. The same periods as above (PreL, Ac-P1/2, Rec-P1/2, PI-P1/2) are separated by vertical dashed lines. The black rectangle (after the primary M1 lesion) represents a period of paralysis for the right hand fingers and progressive (day to day) improvement, during which data are instable, preventing analysis of cumulated daily sessions. The same is true for the left hand (gray rectangle) with respect to the secondary lesion.

as above. Five months following the primary $\mathrm{M} 1$ lesion, a solution of muscimol (GABA inhibitor), accidentally five times over-concentrated, was infused in the hand area of right $\mathrm{M} 1$, inducing a secondary permanent lesion. The functional recovery from this secondary right $\mathrm{M} 1$ lesion was assessed based on the same two behavioral tasks, though during a shorter period (three months), again distinguishing an acute period (Ac-P2), a recovery period (Rec-P2) and a plateau period (PI-P2), with respect to the secondary contralesional forelimb performance (Fig. 1). For both PI-P1 and PI-P2, the plateau onset was quantitatively defined as a period of stable performance during several consecutive daily sessions, as previously reported (Kaeser et al., 2010, 2011): "the onset of the plateau is defined as the first individual data point (total score) for which, among the next 3 individual data points, none exhibits a higher score". The consecutive time windows were distinguished based on the motor performance for each parameter assessed: the score and the contact time (CT) for the modified Brinkman board task and the reaching and grasping times for the modified Klüver board task (see below for detail and Fig. 4).

\section{Behavioral tasks}

Modified Brinkman board task. The modified Brinkman board task has extensively been used in our laboratory (e.g. Liu and Rouiller, 1999; Schmidlin et al., 2004, 2005, 2011; Freund et al., 2006, 2007, 2009; Beaud et al., 2008, 2012; Wannier-Morino et al., 2008; Bashir et al., 2012; Hoogewoud et al., 2013; Kaeser et al., 2010, 2011, 2013, 2014; Chatagny et al., 2013; Wyss et al., 2013). The modified Brinkman board task was performed daily, 5 days a week, except when alternated with ICMS days during the mapping periods and every two days -7 days a week - during the critical acute and recovery periods. The monkey had to freely retrieve bananaflavored pellets from 25 vertical slots and 25 horizontal slots randomly distributed on the board (Fig. 2A). Retrieval from the horizontal slots is more challenging than from the vertical slots, as the horizontal slots require a postural adaptation of the hand in addition to the precision grip itself. For the vertical slots, the precision grip is executed with the hand in its natural position with the tips of the thumb and index finger moving along a vertical axis. In contrast, for the horizontal slots, moving the thumb and index finger tips along the horizontal axis imposed a rotation of the hand which varies depending on the zone of the Brinkman board explored (see Kaeser et al., 2014: their Fig. 5). For instance, when the right hand explored the left half of 
Modified Brinkman board

A

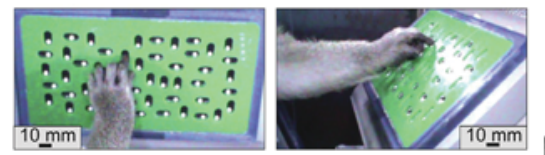

B ${ }_{50}$ Lesion Right hand $\begin{gathered}\text { Lesion } \\ \text { right M1 }\end{gathered}$
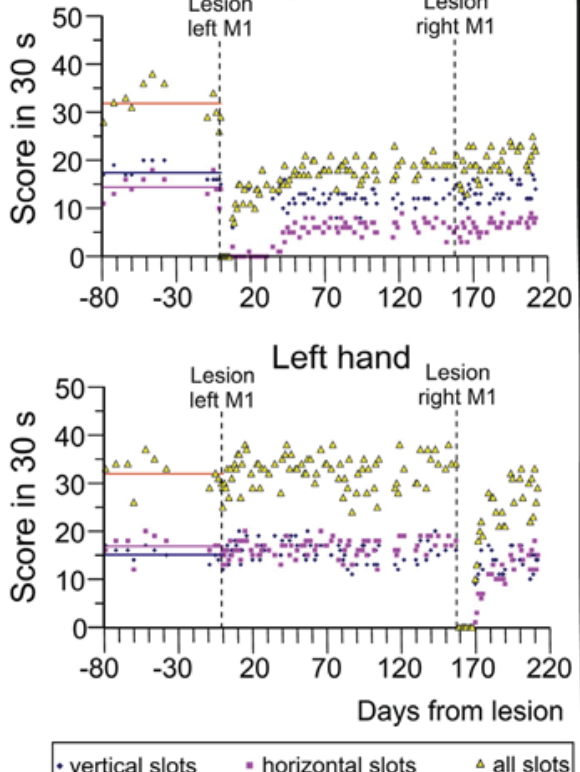

- vertical slots

- horizontal slots

all slots

Modified Klüver board

D
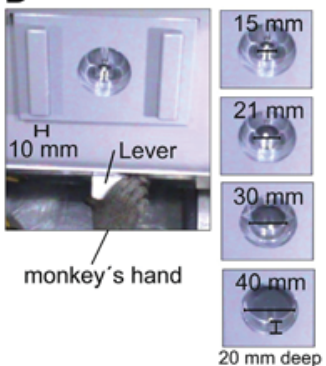

Events intervals
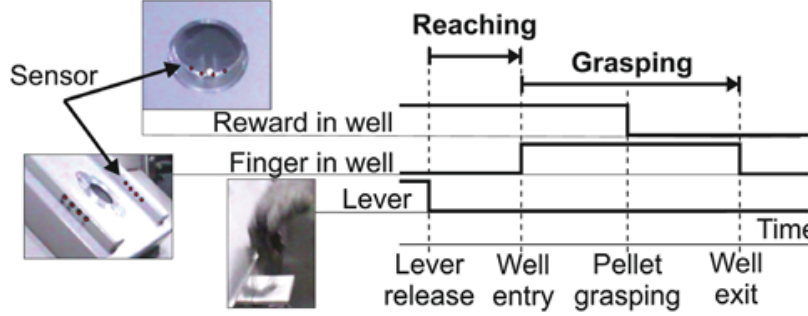

Fig. 2. Behavioral methods. Illustrations of the setups and the motor parameters assessed in the two manual dexterity tasks. The "modified Brinkman board" task: (A) Top and lateral (right) views of the board containing 25 vertical and 25 horizontal slots randomly distributed and placed in front of the monkey. The parameters assessing precision grip performance are the score and the contact time (CT). (B) Illustration of the scores data along the time course of the experiment, separately for the left hand and the right hand, for the vertical slots (blue diamonds), the horizontal slots (purple squares) and the total score (vertical + horizontal slots; yellow triangles). (C) Illustration of the corresponding data for the contact time (mean value and SDs). Values saturated at $3 \mathrm{~s}$ are sessions in which the monkey was unable to perform the task. Pictures illustrate the contact time, corresponding to the time-interval between finger entry and when the pellet is retrieved out of the well, for an individual trial (time frames indicated in ms). The "modified Klüver board" task: (D) Top and lateral (left) views of the board containing four circular wells of different diameter, $15 \mathrm{~mm}, 21 \mathrm{~mm}, 30 \mathrm{~mm}$ and $40 \mathrm{~mm}$, all $20 \mathrm{~mm}$ deep, placed in front of the monkey. On the right, the time-intervals calculated from the on/off waveforms, generated by three series of sensors in the bottom of the wells, at the entry of the wells and on the lever. The two phases of the movement determined by the sensors are: the Reaching phase, from the lever release to the finger's entry in the well, and the Grasping phase, from the finger's entry in the well to the fingers' exit out of the well.

the board, the monkey positioned the index finger tip to the left of the thumb tip along the horizontal axis, a posture achieved by pronation and abduction of the wrist (radial deviation); in contrast, for the right half of the board, the right hand was positioned with the index finger tip at the right with respect to the thumb tip, a posture requiring a supination and adduction of the wrist (ulnar deviation). The monkey had to complete the modified Brinkman board task four times per day, twice per hand, separately and alternatively for the left and the right hand first. Each session was recorded using three cameras positioned around the board, one on the top and two laterally, on the right and on the left, for offline analyses. The manual dexterity performance was quantitatively assessed with the two following parameters (Schmidlin et al., 2011): the score, representing the number of pellets successfully grasped during the first $30 \mathrm{~s}$ (Fig. 2B), and the $\mathrm{CT}$, representing the time of contact between the pellet and the monkey's fingers preceding a successful grasping (Fig. 2C). These two parameters have been shown to be pertinent to assess motor learning as well as functional recovery following motor system injury (Liu and Rouiller, 1999; Schmidlin et al., 2004, 2005, 2011; Freund et al., 2006, 2007, 2009; Chatagny et al., 2013; Wyss et al., 2013; Kaeser et al., 2011, 2014). In order to reduce the impact of possible external disturbances only the session data with the highest total score in $30 \mathrm{~s}$ were taken into consideration for further analyses. Scores were represented on two graphs (Fig. 2B), to visualize the time course of the effects and the recovery following primary $\mathrm{M} 1$ lesion affecting the left hand and then the secondary M1 lesion affecting the right hand. Scores were plotted separately for the vertical slots, the horizontal slots, and the sum of the vertical and horizontal scores. The CT was established for the first five horizontal slots and the first five vertical slots from the same session test used to establish the score for each hand. As for the score, the CT values were plotted for each hand as a function of time (days pre- and postlesion) to assess the effects of the primary and secondary lesion (Fig. 2C). From these analyses, percentages of functional recovery and of performance for score and CT were calculated. These computations were completed separately for the vertical and the horizontal slots, as the movement synergies to 
A Chamber

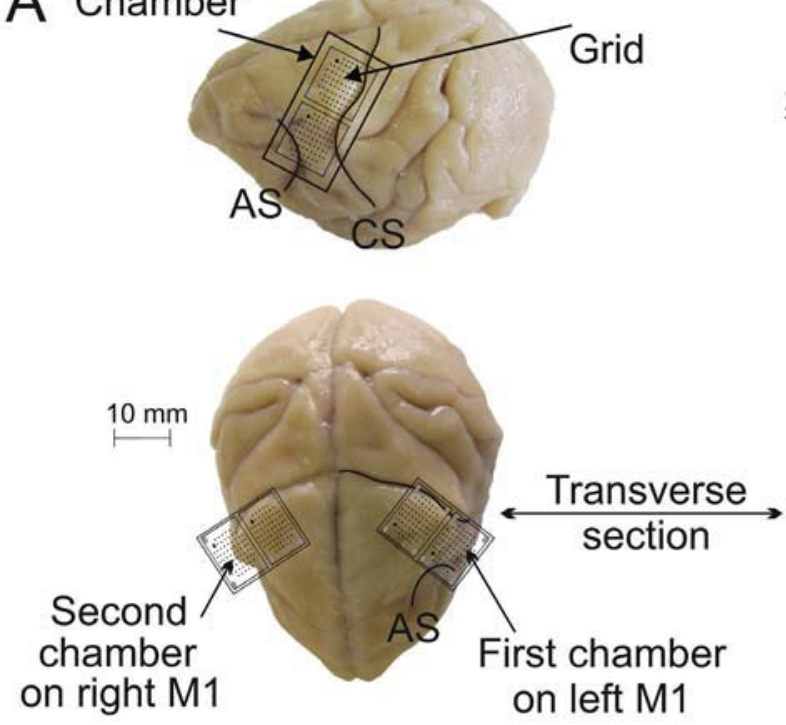

C

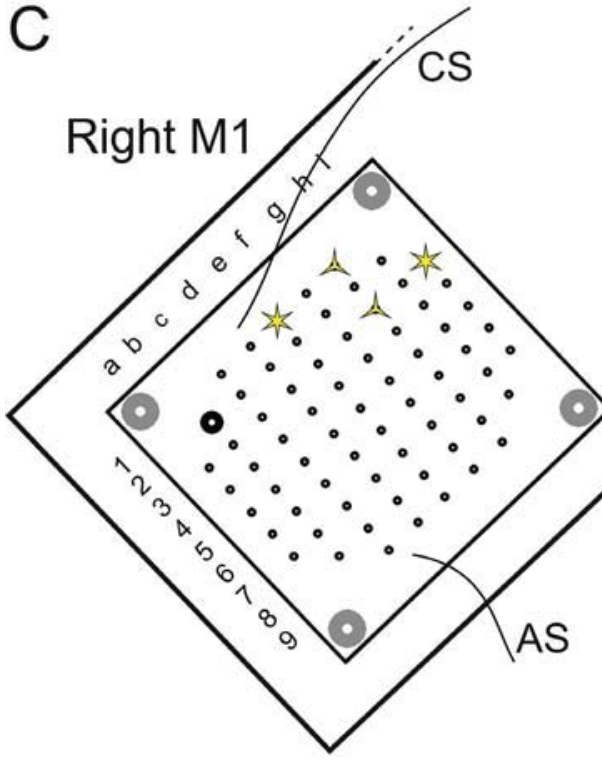

on left M1
B

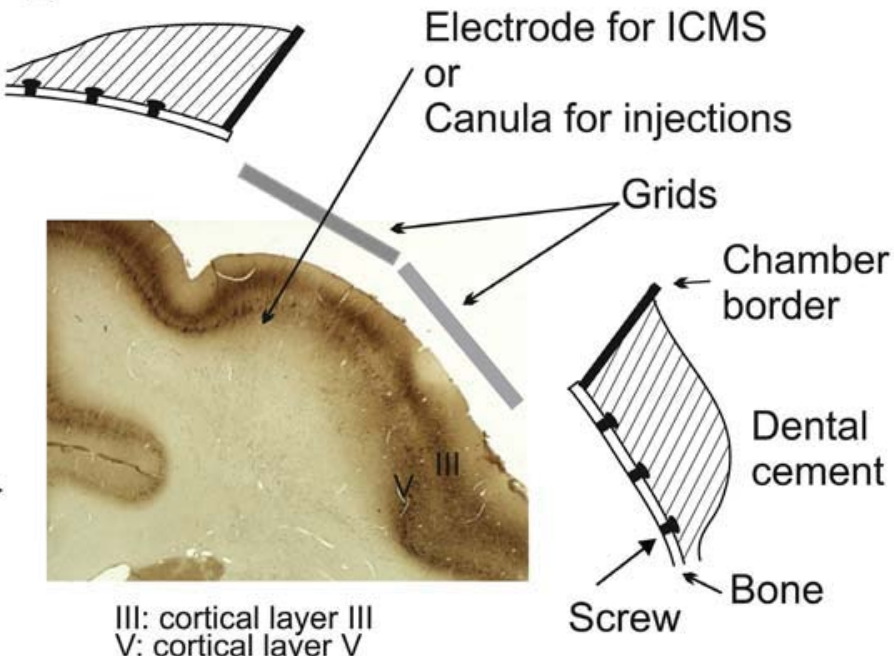

III: cortical layer III
V: cortical layer V

Screw

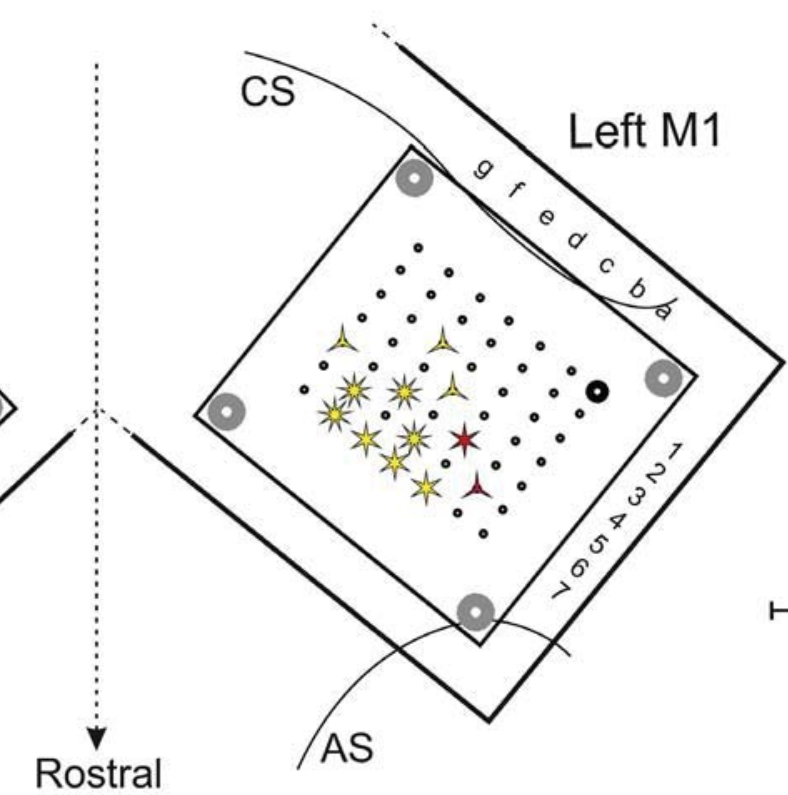

Form:

光 3 sites in depth 2 2 sites in depth A 1 site in depth

Filling:

o Digit

- Wrist

Rostral

\begin{tabular}{|c|c|c|c|c|c|}
\hline & $\mathrm{e}$ & $f$ & $\mathrm{~g}$ & $\mathrm{~h}$ & i \\
\hline 1 & $4-5$ & & 3 & & \\
\hline 2 & & & & & 3-5 \\
\hline 3 & & & 4 & & \\
\hline 4 & & & & & \\
\hline 5 & & & & & \\
\hline 6 & & & & & \\
\hline
\end{tabular}

\begin{tabular}{|r|c|c|c|c|c|c|c|}
\cline { 2 - 8 } \multicolumn{1}{c|}{} & $g$ & $f$ & $e$ & $d$ & $c$ & $b$ & a \\
\hline 1 & & & & & & & \\
\hline 2 & & & & & & & \\
\hline 3 & & & 2 & & & & \\
\hline 4 & & & & 5 & & & \\
\hline 5 & 4 & & $2-4-6$ & & $1-3$ & & \\
\hline 6 & & $2-4-6.5$ & & $0-2-5$ & & 3 & \\
\hline 7 & & $3-5-6.5$ & $3-5$ & $4.5-5$ & $3-5$ & & \\
\hline
\end{tabular}

Fig. 3. Head chronic chamber implants and M1 lesions. Localization of the head chamber implants over the M1 hand area of Mk-DG. (A) Lateral view of the left hemisphere and top view of the brain showing the approximate locations of the grids inserted in the two head chamber implants over the right and left hemispheres. (B) Schematic view of the head chamber implant over a frontal histological section of an unlesioned brain at low magnification, stained for SMI-32. The schematic representation shows the perpendicular penetration of the electrode for ICMS and of the cannula for drug injection in the hole of the epidural grids. (C) Enlarged view of the grids inside the chronic chambers, giving access to M1. The sites of injection of ibotenic acid in the left M1 and muscimol in the right M1 are indicated with stars, as indicated in the inset on the right. The color code indicates the body part movements elicited during the ICMS sessions (yellow for the fingers and red for the wrist). CS: Central Sulcus, ArcS: Arcuate Sulcus. Below, tables indicate the depth of infusion sites (in $\mathrm{mm}$ ). Along some penetrations, the drug was infused at multiple sites, corresponding to different depths (2 or 3 depths). 

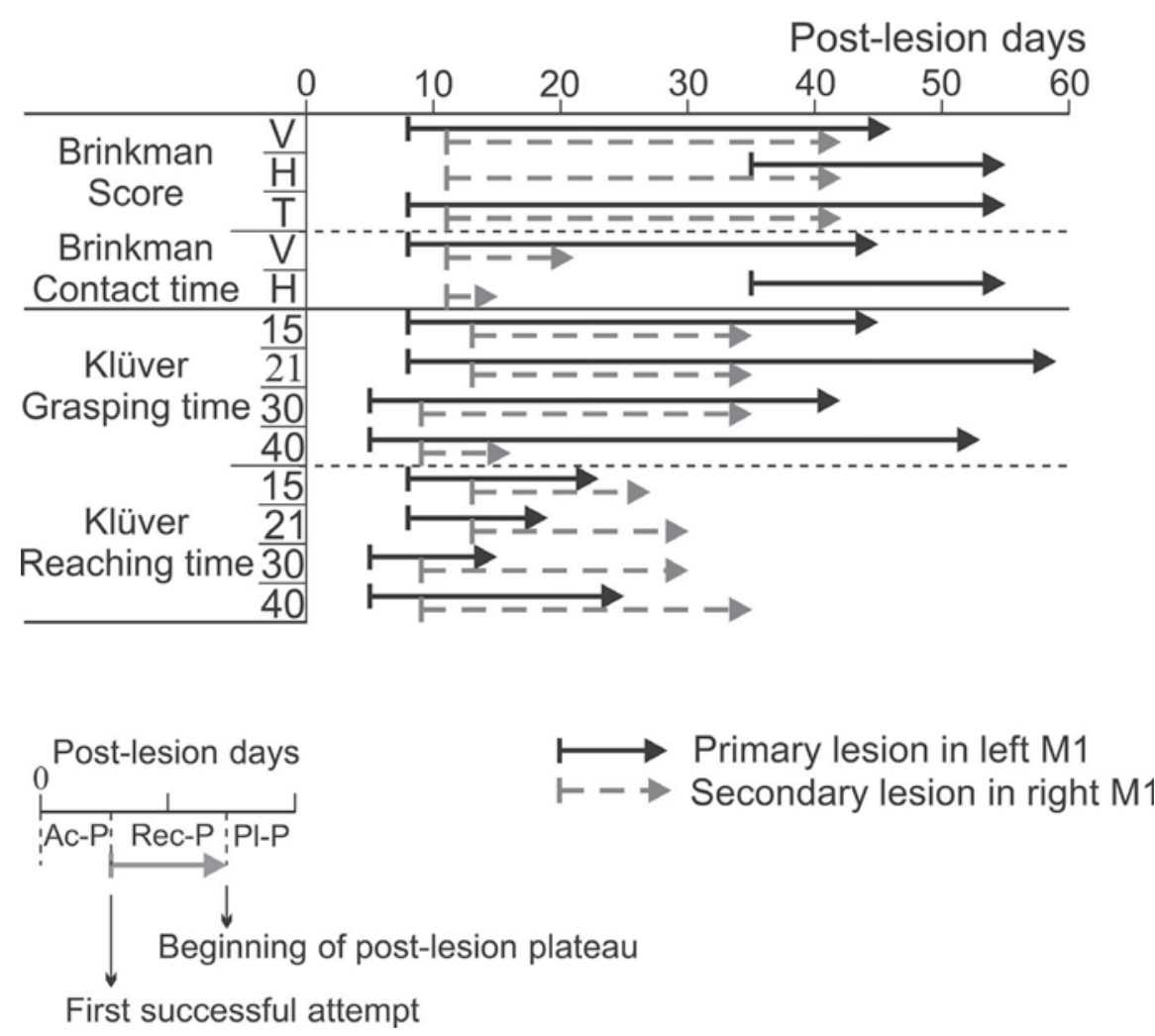

Fig. 4. Time windows of post-lesion functional recovery for the two behavioral tasks. Recovery time-intervals (days) for the respective contralateral hand following the primary lesion of the left M1 hand area (solid black arrows) and the secondary lesion of the right M1 hand area (dashed gray arrows). The length of the arrow is a representation of the recovery period (Re-P) durations from the first day post-lesion of a successful attempt to the first day of the plateau for the modified Brinkman board task parameters (score and contact time) and for the modified Klüver board task time parameters (grasping and reaching phases). The time-interval between the day of the lesion (day 0 ) and the left extremity of the arrow is the period during which the subject was totally unable to perform the task (acute period: Ac-P). The plateau period (PI-P) starts from the head of the arrow. V: vertical slots; $\mathrm{H}$ : horizontal slots; T: vertical and horizontal slots mixed; 15, 21, 30 and 40 for the four well diameter sizes (in $\mathrm{mm}$ ).

retrieve pellets from the horizontal slots involved more complex movements (see above). Functional recovery was calculated for the contralesional hands, respective to the primary or secondary lesion, by comparing the pre-lesion and post-lesion performances at plateau. For the score, the percentage of recovery was given by the performance at post-lesion plateau (PI-P1 or PI-P2) divided by the performance pre-lesion (PreL) ${ }^{*}$ 100: (PIP/PreL $\left.{ }^{*} 100\right)$. As the CT increases in case of reduced performance, the percentage of recovery was given by the CT pre-lesion (PreL) divided by the CT post-lesion $(\mathrm{PI}-\mathrm{P} 1$ or PI-P2) * 100: (PreL/PI-P * 100). Moreover, percentages of performance were calculated for the ipsilesional hands, at three periods with reference to each of the primary lesion and the secondary lesion, respectively: for score the values are given by Ac-P/PreL * 100 , Rec-P/ $\mathrm{PreL}^{*} 100, \mathrm{PI}-\mathrm{P} / \mathrm{PreL}{ }^{*} 100$; for $\mathrm{CT}$ the values are given by PreL/Ac-P * 100, PreL/Rec-P* 100, PreL/PI-P ${ }^{*} 100$. For comparisons of the scores and of the CT, statistical analyses were performed using one-way ANOVA and the multiple comparisons Holm-Sidak method for post hoc analyses (SigmaStat, Systat Software, San Jose, CA, USA).
Modified Klüver board task. The modified Klüver board task was performed every second days, 2-3 times a week, except when ICMS testing occurred as described above. During the critical acute and recovery periods behavioral testing took place every other day, 7 days a week. The monkey had to retrieve pellets from four wells of different diameters $(15 \mathrm{~mm}, 21 \mathrm{~mm}, 30 \mathrm{~mm}$ and $40 \mathrm{~mm}$ of diameter, all $20 \mathrm{~mm}$ deep), filled automatically after a random delay $(0.5,1,1.5$ or $2 \mathrm{~s})$ upon pressing on a lever (Fig. 2D). The four progressively increasing diameters were designed to involve 1, 2, 3 and 4 fingers respectively, in addition to the thumb for grasping. The monkey performed 50 trials per diameter, starting with the $15 \mathrm{~mm}$ up to the $40 \mathrm{~mm}$ diameter, separately and alternatively with the left or the right hand first. For automatic recordings of behavioral time parameters, the experimental setup was designed with sensors located at three sites (Fig. 2D): (1) On the start lever to detect hand pressure and release. (2) A series of sensors at the well entry. (3) A series of sensors at the bottom of the well. Based on these detectors, two different phases of the task were analyzed: the reaching phase from the time point the monkey released the lever to the moment a finger entered in the well and the grasping phase during which the fingers were in the well to grasp the pellet (Fig. 2D). Signals from sensors were digitized and processed with CED 1401 interface using Spike 2 software (Cambridge Electronic Design, Cambridge, UK). The corresponding on/off signals were analyzed offline with Matlab R2012b (Mathworks Inc., Natick, MA, USA) to determine reaching and grasping times for each trial. Error trials, removed for the offline analysis, were defined as pellets fallen or not picked at all, more than one pellet in the well, and when the monkey was disturbed by external distracting events. In addition, specific criteria were defined to reject reaching time values from analysis, such as trials in which the lever was released before the pellet arrived in the well, as well as trials in which reaching time values exceeded $500 \mathrm{~ms}$. Reaching time values lower than $500 \mathrm{~ms}$ represented more than $99 \%$ of all values and those higher than $500 \mathrm{~ms}$, and representing less than $1 \%$ of all values, were considered as biased by external interferences. In cases where several grasping attempts were needed to collect a pellet, all individual grasping time values were added, yielding a single sum trial value. Similarly as for the 
A Right hand
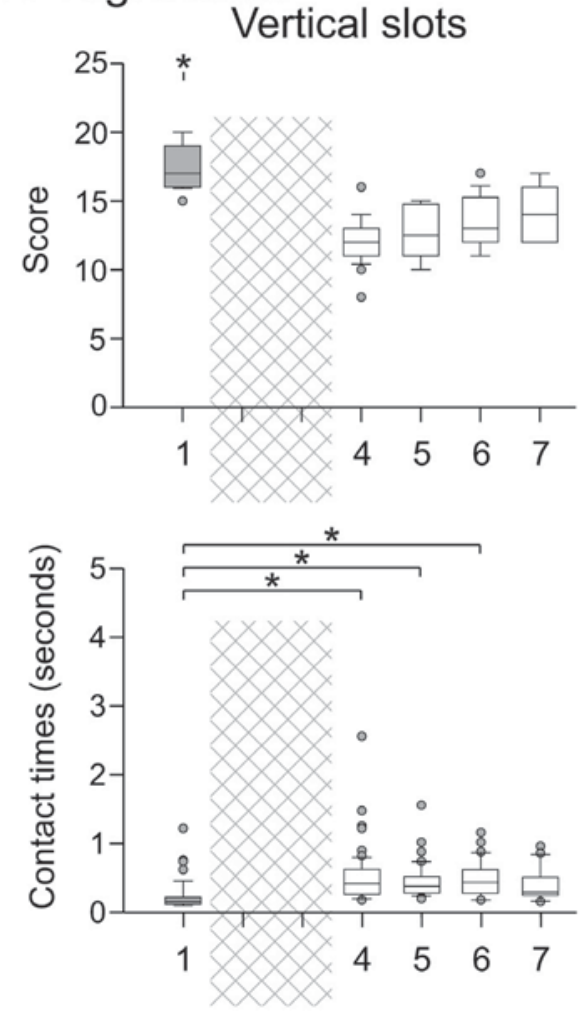

\section{$B$ Left hand}
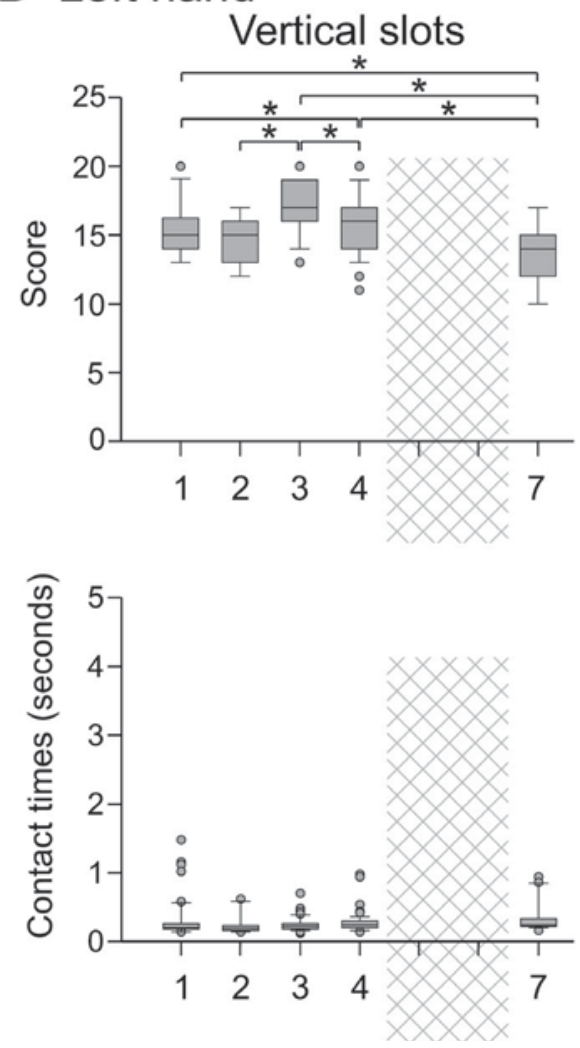

modified Brinkman board task (see above), percentages of functional recovery were calculated for both reaching and grasping times, separately for the contralesional hands and ipsilesional hands, with reference to the primary and secondary lesions.

Alongside the automatic recording of the time intervals, the reaching properties and the grasping strategies were assessed (Figs. 6 and 7), based on offline analysis of video sequences recorded using three fixed cameras (50 frames per seconds). One camera filmed the well of the board from above; whereas two cameras placed laterally (one on each side) captured the two dimensional (2D) trajectory of the hand as well as the distance between the finger tips (see below). The cameras and the primate chair occupied the same positions from one daily session to the next. The preshaping properties during the reaching phase were assessed from the first five successful trials of 3-10 sessions within each time window (as defined in Fig. 1). Pre-shaping was assessed by measuring the distance between the tip of the thumb (D1) and the tip of the index finger (D2), both moving along a 2D trajectory perpendicular to the axis of the corresponding camera placed laterally: left side camera for the right hand and right side camera for the left hand.

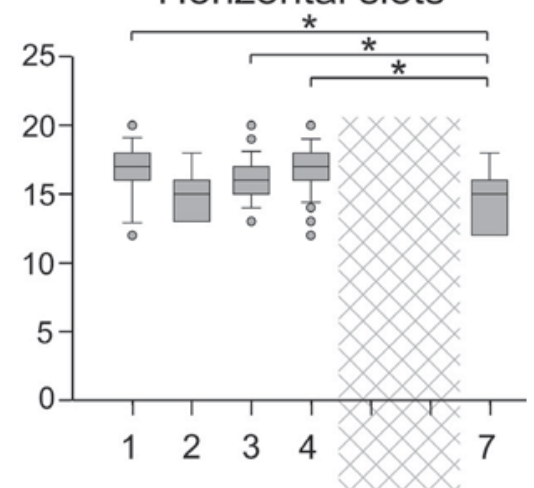

Fig. 5. "Modified Brinkman board task": effects of the primary and secondary lesions. Scores and contact times (CT), for the right hand $(A)$ and for the left hand (B), shown for the consecutive time windows of the perilesional periods (as defined in Fig. 1). The consecutive time windows are indicated by numbers along the abscissa: $1=$ PreL, $2=$ Ac-P1, 3 = Rec-P1, $4=$ PI-P1, 5 = AcP2, $6=$ Rec-P2 and $7=$ PI-P2. The periods immediately following the primary lesion (in A) and the secondary lesion (in B) are not depicted by a box plot as they correspond to a total inactivation (Ac-P) and a progressive (instable) recovery (Rec-P) of the contralesional hand and were therefore indicated by a rectangle grid zone. On the box plots, the median and all outlier values are represented, in addition to the 10, 25, 75 and 90 percentiles. The white boxes emphasize the comparison between PI-P1 and the subsequent periods after the secondary lesion for the right hand (see text). "For statistically significant differences $(p<0.05)$; when positioned only above a single box, it means that this set of values is statistically significantly different from all the other sets of values of the graph. 


\section{A Right hand}

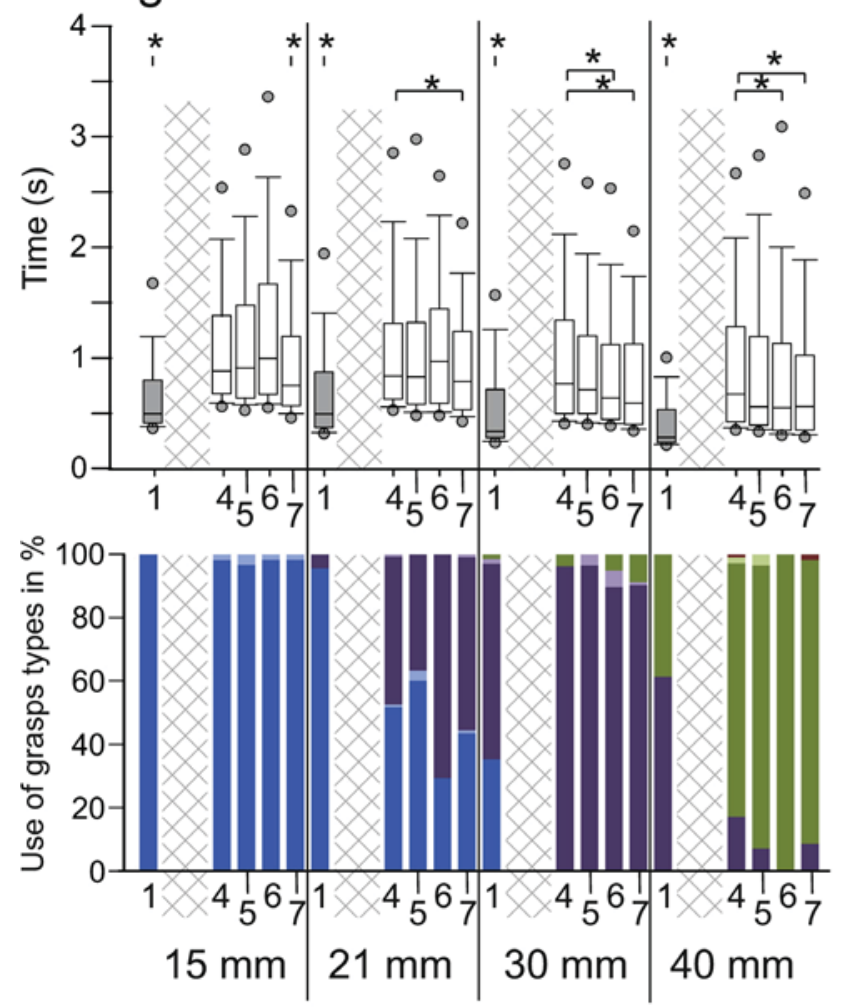

\section{B Left hand}

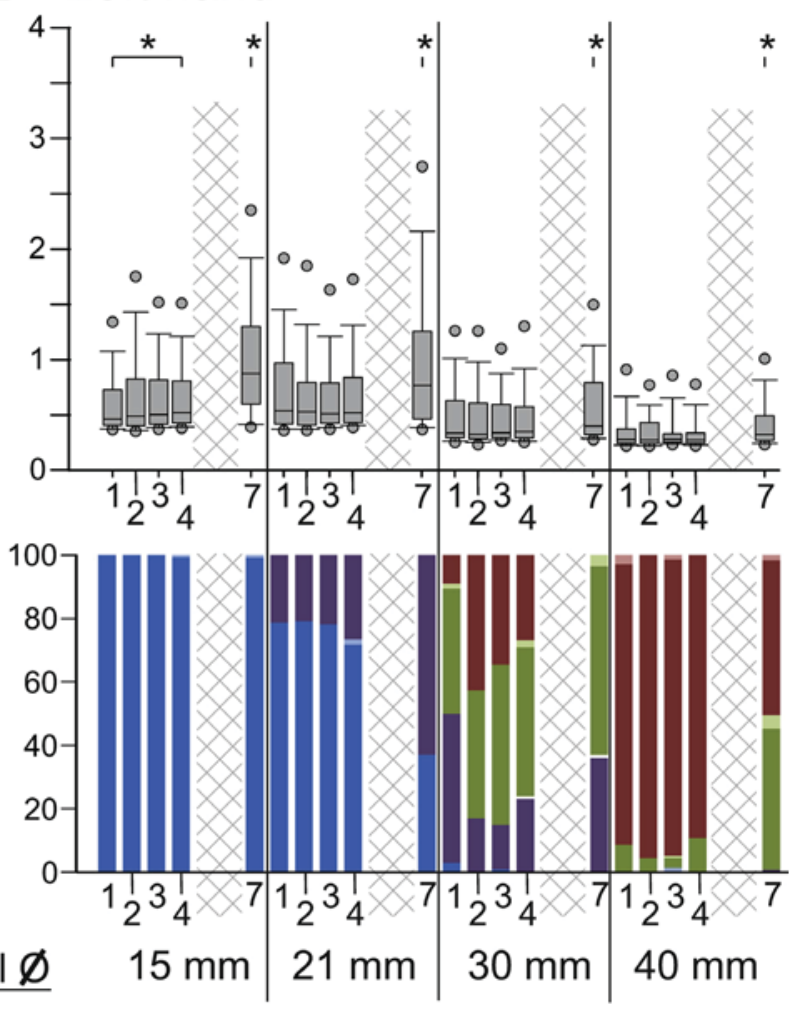

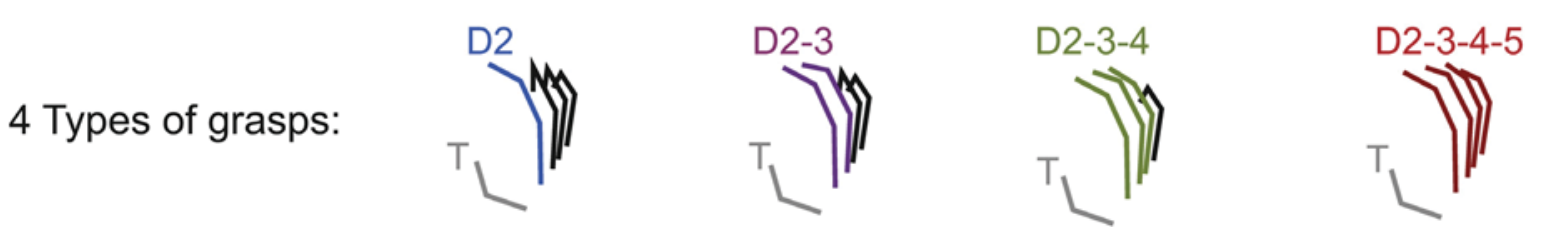

Fig. 6. "Modified Klüver board task": effects of the primary and secondary lesions during the grasping phasein each panel (A for the right hand and $B$ for the left hand), the top box plots show grasping time interval values whereas the distribution of finger use strategies is shown in the bottom bar graphs. The consecutive time windows are indicated by numbers along the abscissa: $1=\mathrm{PreL}, 2=\mathrm{Ac}-\mathrm{P} 1,3=\mathrm{Rec}-\mathrm{P} 1,4=\mathrm{Pl}-\mathrm{P} 1,5=\mathrm{Ac}-\mathrm{P} 2$, $6=$ Rec-P2 and $7=$ PI-P2. The grasping time intervals in box plots are as in Fig. 5, except that all outlier values were replaced by the 5th/95th percentiles. The white boxes emphasize the comparison between PI-P1 and the subsequent periods after the secondary lesion for the right hand (see text). "For statistically significant differences $(p<0.05)$; when positioned only above a single box, it means that this set of values is statistically significantly different from all the other sets of values of the graph. The grasping strategies are represented in the form of bar graphs by the respective percentage of trials performed with the various finger configurations, either successful (dark color) or failed (light color). Below the bar graphs, legend of the color code corresponding to the combination of fingers used for grasping. Results are represented for each of the peri-lesions time-intervals (see Fig. 1), except for the periods of total inactivation (Ac-P) and progressive unstable recovery (Rec-P) indicated by a shaded rectangle. All data are represented separately for each well diameter.

The interdigit tip distance was measured manually, trial by trial and frame by frame (using the software Dartfish ${ }^{\circledR}$; http://www. dartfish.com to visualize the video sequences). Irrespective of the number of fingers (D2/ D3/D4/D5) involved for the grasping, D2 was invariably involved to retrieve pellets from the well. Although the distances between D1 and D2 were measured in each video frame, the trajectory between the start lever and the well entry was divided in three different zones (Fig. 7): (i) the initial zone just after the monkey released the lever and initiated the reaching movement (corresponding to the movement trajectory occurring at distances greater than $9 \mathrm{~cm}$ from the well), (ii) the intermediate zone of the trajectory along which the hand is moved toward the board (ranging from 3 to $9 \mathrm{~cm}$ away from the well); (iii) the terminal zone (up to $3 \mathrm{~cm}$ away from the well), where the monkey prepared its finger(s) to enter the well.

The grasping strategy (during the pellet retrieval) was assessed for the 25-30 first trials of 2-6 sessions in each time period of the protocol as defined in Fig. 1. This grasping strategy was defined as the number of fingers used to retrieve the pellet. The monkey used four different finger configurations for grasping: D2 or D2 $+\mathrm{D} 3$ or $\mathrm{D} 2+\mathrm{D} 3+\mathrm{D} 4$ or $\mathrm{D} 2+\mathrm{D} 3+\mathrm{D} 4+\mathrm{D} 5$ (Fig. 6), corresponding to the number of fingers inside the well at the time of the pellet retrieval. Successful and failed trials were identified for each finger configuration and expressed in percentages.

Reaching properties and grasping strategy were not analyzed for the contralesional hand, with reference to the primary and secondary lesion during periods of 


\section{A Right hand}

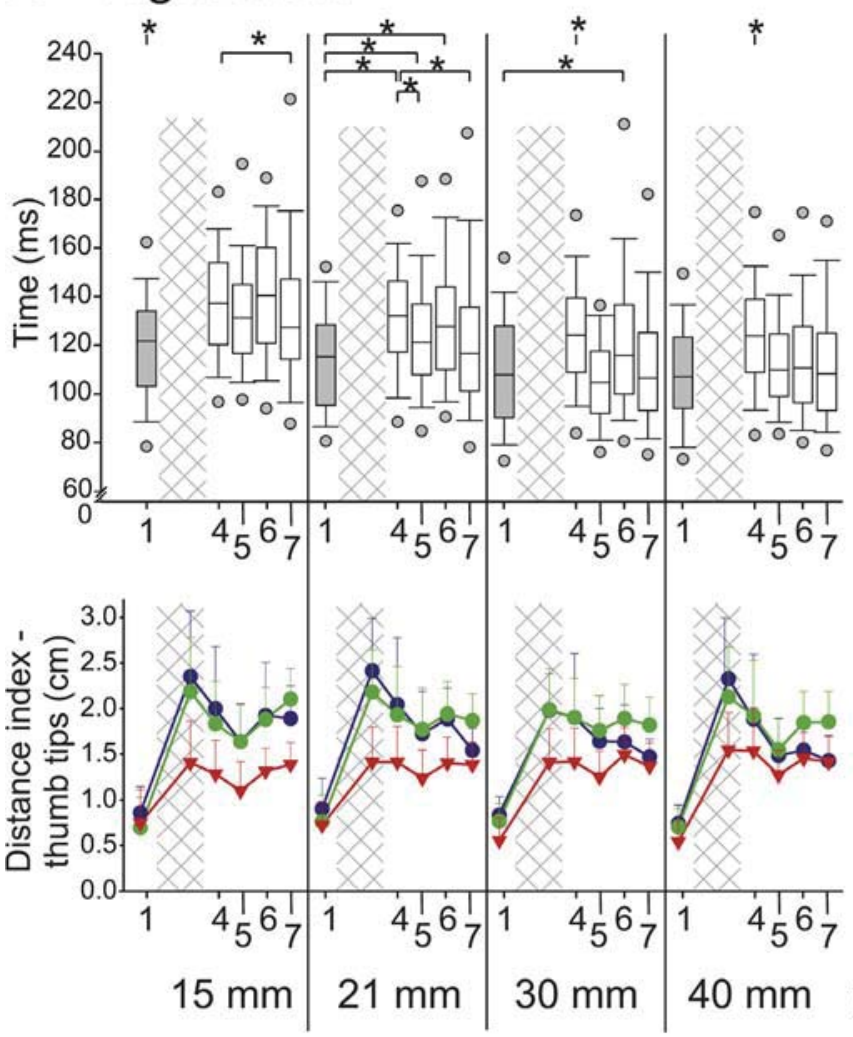

B Left hand

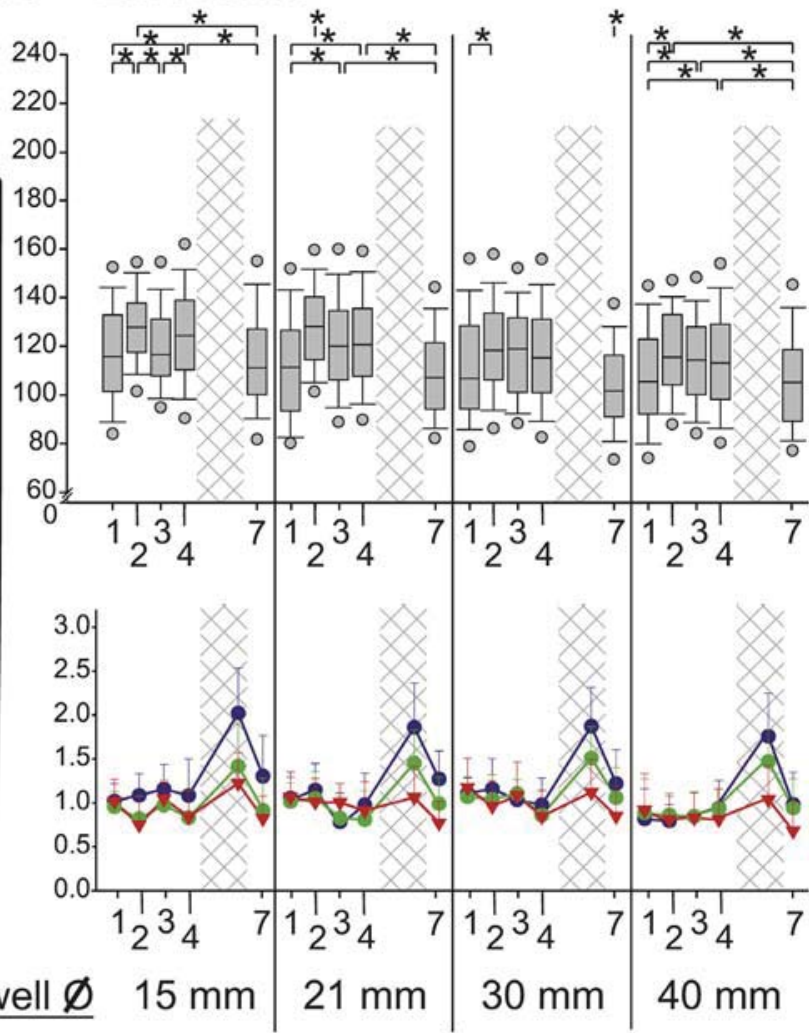

\section{Movement zones for reaching properties :}

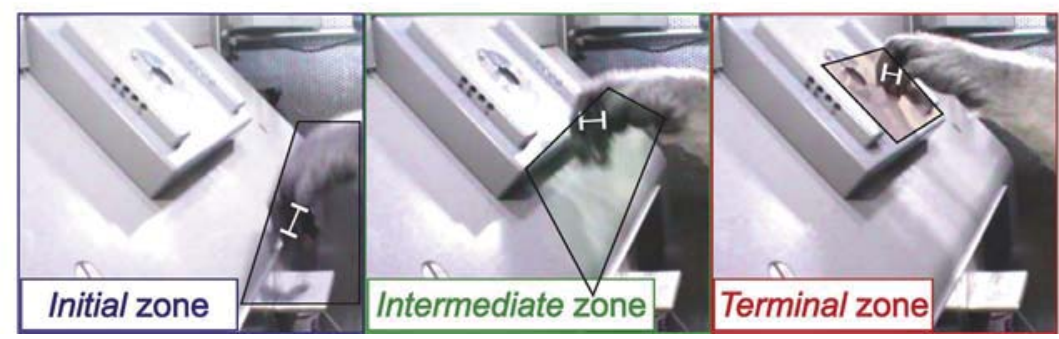

Fig. 7. "Modified Klüver board task": effects of the primary and secondary lesions during the reaching phase in each panel (A for the right hand and $\mathrm{B}$ for the left hand), the reaching time values and reaching strategies (interdigit distance) are displayed. Same conventions as in Fig. 6 for the reaching times results. The consecutive time windows are indicated by numbers along the abscissa: $1=\mathrm{PreL}, 2=\mathrm{Ac}-\mathrm{P} 1,3=\mathrm{Rec}-\mathrm{P} 1,4=\mathrm{Pl}-$ $\mathrm{P} 1,5=\mathrm{Ac}-\mathrm{P} 2,6=\mathrm{Rec}-\mathrm{P} 2$ and $7=\mathrm{Pl}-\mathrm{P} 2$. The reaching properties are represented by the multiple straight lines graphs showing means and standard deviations of the distances between the index and thumb tips collected in the three zones of interest along the $2 \mathrm{D}$ movement trajectory: initial zone (blue), intermediate zone (green) and terminal zone (red) as illustrated in the bottom panel (see text).

instable and increasing performance, such as Ac-P and Rec- $P$, but only for the ipsilesional hand during these time windows. Statistical analyses were performed using non-parametric ANOVA and Dunn's test for post hoc analysis (SigmaStat, Systat Software, San Jose, CA, USA).

\section{Surgery}

Anesthesia for surgical procedures was described in detail in a recent report from this laboratory (Lanz et al., 2013). Sedation was induced with a mixture of ketamine

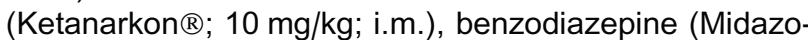
lam; $0.1 \mathrm{mg} / \mathrm{kg}$; i.m.) and methadone (0.2 mg/kg; i.m.).
Following induction, Atropine $(0.05 \mathrm{mg} / \mathrm{kg}$, i.m.), Carprofen (Rimadyl ${ }^{\circledR} ; 50$ mg/ml; 4 mg/kg; i.m.), Dexamethasone (Decadron $\AA ; 0.3 \mathrm{mg} / \mathrm{kg}$ diluted $1: 1$ in saline; i.m.) and antibiotic, Synulox (Amoxicillin-Clavulanic Acid; $8.75 \mathrm{mg} / \mathrm{kg}$; s.c.) were administered. Deep anesthesia was maintained with the combination of an intravenous perfusion of propofol (diisopropylphenol; $1.2-3.6 \mathrm{mg} / \mathrm{kg} /$ h diluted 1:2 in Ringers solution) and an anesthetic gas (Sevoflurane; $2.5 \%$; 0.5-1 L/min) mixed with a 50/50 mixture of $\mathrm{O}_{2}$ and air delivered via a tracheal cannula. During the entire surgery, the animal was continuously perfused with Ringer-lactate $(5 \mathrm{ml} / \mathrm{kg} / \mathrm{h}$; i.v.). A drop of $(\sim 0.5 \mathrm{ml}$, $1 \%$ ) lidocaine was introduced into the larynx prior to intubation, and incision sites were also treated with lidocaine 
$1 \%$; s.c. Additionally prior to potentially painful steps (e.g. craniotomy), opioid analgesia was administered (Fentanyl; $0.1 \mu \mathrm{g} / \mathrm{kg} / \mathrm{min}$; i.v.). Pain and infections following surgery were prevented by postoperative treatment with Carprofen and antibiotic Synulox during the ten following days. All surgeries were performed in a facility under sterile conditions and approved by the (Swiss) cantonal veterinary office. During the entire surgical procedure, body temperature, $\mathrm{O}_{2}$ saturation, heart rate, ECG, respiration rate, exhaled $\mathrm{CO}_{2}$ were continuously monitored, allowing adjustment of the flow of anesthetic agents (gas and propofol perfusion flows) to maintain normal physiological parameters.

\section{Head chamber implants}

Chronic head chamber implants were designed as described in a previous report (Schmidlin et al., 2008) in order to access the M1 hand area (Fig. 3). Two identical chambers were implanted successively over the left and right hemispheres (Fig. 3A). The chambers were designed to hold two Tecapeek grids $(11 \mathrm{~mm} \times 11 \mathrm{~mm} \times 3 \mathrm{~mm})($ Fig. $3 \mathrm{~A}$ and B). For electrode and cannula penetrations, the two grids were perforated in an $8 \times 8$ configuration, with holes separated by $1 \mathrm{~mm}$ (Fig. 3B and C). Under deep anesthesia, the head of the monkey was fixed in a stereotaxic head holder. Using stereotaxic coordinates supplemented with MRI scans, the two grids for the first chamber on the left hemisphere were centered above $\mathrm{M} 1$, the first positioned at $15 \mathrm{~mm}$ lateral and $15 \mathrm{~mm}$ anterior and the second positioned at $20 \mathrm{~mm}$ lateral and $20 \mathrm{~mm}$ anterior. The two grids of the second chamber on the right hemisphere were centered above M1 hand area, the center of the first grid positioned at $17 \mathrm{~mm}$ lateral and $17 \mathrm{~mm}$ anterior and the second positioned at $22 \mathrm{~mm}$ lateral and $24 \mathrm{~mm}$ anterior. A skull window of the internal chamber dimensions $(25 \mathrm{~mm}$ length and $12 \mathrm{~mm}$ width) was opened over the corresponding coordinates. The chamber was adjusted on the window's borders with an inclination of $30^{\circ}$ to the horizontal plane (approximately parallel to the brain surface) and was cemented (dental acrylic) along with four to six self tapping titanium screws anchored to the adjacent skull. The two grids were positioned epidurally and fixed on the internal border of the chamber allowing guidance of electrodes and cannula perpendicularly to the cortical surface (Fig. 3B).

\section{Motor cortex lesions}

Mapping of the hand area in M1 was performed prior to left and right M1 lesions. Mapping was achieved using intra-cortical microstimulation (ICMS) in order to localize the extent of digit representation for the subsequently lesioned hand area on the precentral surface and in the wall of the central sulcus in M1. ICMS was performed twice a week under light sedation (ketamine $4 \mathrm{mg} / \mathrm{kg}$; medetomidine $0.04 \mathrm{mg} / \mathrm{kg}$; co-injected i.m.). Tungsten microelectrodes with typical impedances between 0.1 and 1.0 M $\Omega$ (Frederick Haer \& Co., Bowdoinham, ME, USA) were used for ICMS. Electrodes were manually inserted at the selected grid locations starting approximately $2 \mathrm{~mm}$ below the pial surface and advanced, generally by steps of $1 \mathrm{~mm}$ to a maximal final depth of $8 \mathrm{~mm}$ when targeting the rostral bank of the central sulcus (Kaeser et al., 2010). ICMS consisted of six biphasic pulses ( $0.2 \mathrm{~ms}$ duration) delivered in $30 \mathrm{~ms}$ trains at a sweep rate of $0.5 \mathrm{~Hz}$. Before starting the ICMS session, medetomidine, a muscular relaxant, was partially reversed by injection of atipamezol (Alzane ${ }^{\circledR} 0.05 \mathrm{mg} /$ $\mathrm{kg}$ ), a medetomidine antagonist. Light anesthesia was maintained by injection of $0.05 \mathrm{ml}$ of ketamine each $4 \mathrm{~min}$ till the end of the session. Due to the absence of chronic EMG electrodes, one experimenter carefully observed the contralateral forearm (also other territories like face, neck, trunk, etc) to detect movement or small muscle twitch. Furthermore, the same experimenter held the monkey's forelimb in order to feel for muscle twitches/movements and to manipulate the posture of the forelimb, as ICMS results can vary according to rest position. In parallel, a second experimenter adjusted the intensity of the ICMS current. The body part movements elicited, depth and intensity threshold of each ICMS site were collected. At this step, an additional dose of atipamezol was injected to complete the medetomidine reversion (Alzane ${ }^{\circledR} 0.2 \mathrm{mg} / \mathrm{kg}$ minus the first injection of $0.05 \mathrm{mg} / \mathrm{kg}$ ) and the monkey was monitored up to recovery from the anesthesia.

The two M1 lesions were chemically induced by drug injection with a $10-\mu \mathrm{l}$ Hamilton microsyringe connected to a cannula, targeting the digit representation of the hand area. The cannula was manually inserted and advanced through the grid holes selected from the ICMS sites eliciting digit movements at low ICMS intensities, characteristic of fast conducting and low threshold corticomotoneuronal M1 neurons (Wyss et al., 2013) (Fig. 3C). The primary permanent lesion of the left M1 hand area was achieved by infusions of the excitotoxic ibotenic acid 95\% (Sigma \#I-2765, $10 \mu \mathrm{g} / \mu \mathrm{l}$ in phosphate buffered saline) at a volume of $1 \mu \mathrm{l}$ at each of 24 sites (Fig. 3C). Initially aimed to be reversible and more restricted, the secondary lesion of the right $\mathrm{M} 1$ hand area was achieved with microinjections of the reversible GABA agonist muscimol (Sigma \#M-1523, $5 \mu \mathrm{g} / \mu \mathrm{l}$ in saline buffer), at a volume of $1 \mu \mathrm{l}$ at each of seven sites (Fig. $3 \mathrm{C}$ ). It turned out that the concentration of muscimol was accidentally overdosed $(5 \mu \mathrm{g} / \mu \mathrm{l}$ instead of $1 \mu \mathrm{g} / \mu \mathrm{l})$, leading to a long lasting deficit (instead of a reversible deficit of one day at most). In order to cover the maximal surface of the digit representation among the hand area, adjacent sites of injection were spaced by $1-3 \mathrm{~mm}$, in line with the diffusion of the substance established at $1.5 \mathrm{~mm}$ for muscimol (Martin, 1991) and up to $3 \mathrm{~mm}$ for ibotenic acid (Murata et al., 2015).

After reaching the plateau of behavioral performances, 17 weeks and 3 days after the primary lesion, a second MRI scan was performed to localize the lesion and its extent, following a similar procedure as the first MRI scan during the PreL (see also Peuser et al., 2011). The MRI acquisitions were done under anesthesia, induced with a mixture of ketamine (Ketasol ${ }^{\circledR}$ $10 \mathrm{mg} / \mathrm{kg}$; i.m.) and benzodiazepine (Midazolam; 
$0.1 \mathrm{mg} / \mathrm{kg}$; i.m.), then maintained with a flow of a mixture of propofol (diisopropylphenol 1\%; 1.2-3.6 mg/kg/h diluted $1: 1$ in Ringer; i.v.) and ketamine (Ketasol ${ }^{\circledR}$ $3.75 \mathrm{mg} / \mathrm{kg} / \mathrm{h}$; i.v.). The head of Mk-DG was fixed in a plastic stereotaxic headholder and the monkey was placed in ventral position for MRI acquisitions. The volume of the lesion was calculated on a parasagittal T2 Flair Cube 3D acquisition of the full head (TE $=140.1$; $\mathrm{TR}=6000 ; 800 \mu \mathrm{m}$ slice thickness) acquired on a Discovery MR750 3.0T scanner (GE Medical System; Cantonal Hospital of Fribourg-Switzerland). The volume was estimated with the Cavalieri method using areas of region of interest surrounding the lesioned area and measured with Osirix software (http://www.osirix-viewer.com).

\section{Histology}

At the end of the experiments, the monkey Mk-DG was deeply anaesthetized with ketamine and received a lethal dose of sodium pentobarbital $(60 \mathrm{mg} / \mathrm{kg}$; i.v.). The monkey was perfused with saline $(300 \mathrm{ml})$ followed by fixative (paraformaldehyde $(4 \%)$ in $0.1 \mathrm{M}$ of phosphate buffer $(\mathrm{pH}=7.6))$, followed by solutions of increasing concentrations of sucrose $(10 \%, 20 \%$ and $30 \%$; for detail see e.g. Wannier et al., 2005; Beaud et al., 2008). The brain and spinal cord were extracted and immersed in a solution of sucrose $(30 \%$ in phosphate buffer, $\mathrm{pH}=7.6)$. For anatomical reconstruction of the $\mathrm{M} 1$ lesions, the brain was sectioned in $50 \mu \mathrm{m}$ thick coronal sections. Out of five series of sections, one series was Nissl stained with Cresyl Violet and a second series was labeled with the SMI-32 marker as already described in previous reports (Beaud et al., 2008, 2012; Wyss et al., 2013). In more detail, under light microscope, Neurolucida software was used to draw contours delineating the cortical and subcortical lesion sites (Wyss et al., 2013). On consecutive SMI-32 labeled sections, the cortical lesion was delimited based on cortical layer $\mathrm{V}$ interruption, the lesion site representing the cumulated volume of regions where the cortical layer $\mathrm{V}$ was deprived of SMI-32positive pyramidal neurons. On consecutive Nissl stained sections, the subcortical lesion was delimited by surrounding the necrotic tissue in the white matter. Using Neurolucida software, contours were used to calculate the volumes of the cortical lesions (in $\mathrm{mm}^{3}$ ) based on the Cavalieri method (e.g. Pizzimenti et al., 2007; Wyss et al., 2013).

\section{RESULTS}

For the modified Brinkman board task, Kaeser and colleagues $(2010,2014)$ have shown no preference to retrieve the less challenging vertical slots first before the lesion. On the contrary, they have shown that the intact monkey retrieved pellets from the vertical and horizontal slots according to a mixed temporal sequence. In other words, there was no bias toward vertical slots, which may have occurred due to fatigue if horizontal slots were visited later. The lack of slot orientation preference in the intact monkey was no longer present after unilateral lesion of $\mathrm{M} 1$, especially during the acute recovery period, during which vertical slots were preferred and more successful.

\section{Recovery time course for the two M1 lesions}

The various motor parameters, derived from the two behavioral tasks, were affected differently by the primary and secondary lesions. This was firstly observed in the duration of inability to perform grasping (Ac-P), from the lesion until the day of the first successful attempt to reuse the contralesional hand for grasping. Secondly, the duration of functional recovery period (Rec-P) was assessed, beginning from the first successful re-use attempt to the beginning of the post-lesion plateau (Fig. 4). Generally, Mk-DG started to recover earlier after the primary $\mathrm{M} 1$ lesion (solid black arrow) than after the secondary M1 lesion (dashed gray arrow). Contrarily, the duration of the recovery period was shorter after the secondary M1 lesion as far as the parameters assessing the grasping aspect in both tasks are concerned, whereas the recovery of reaching was shorter after the primary M1 lesion (Fig. 4).

Following the primary lesion of the left M1 hand area (solid black arrows in Fig. 4), as expected, the less challenging grips in wells with larger diameter recovered earlier than the more challenging ones. In the modified Klüver board task, recovery started with the power grip in the large wells (30 and $40 \mathrm{~mm}$ ), followed by precision grip in the small wells (15 and $21 \mathrm{~mm}$ ). Later steps of recovery involved the vertical slots of the modified Brinkman board task, and finally the complex movements of precision grip in a pro-supination position required for the horizontal slots of the modified Brinkman board task. A fairly comparable sequence of recovery was observed after the secondary lesion of the right $\mathrm{M} 1$ hand area for the Klüver board task (dashed gray arrows in Fig. 4).

\section{Contribution of the intact M1 to the functional recovery from the primary unilateral M1 lesion}

The description of the behavioral data below is focused on the extent of functional recovery from the primary M1 lesion for the various motor parameters assessed with the two manual dexterity tasks, and whether the secondary lesion affected the recovered performance. To this latter aim, the performance at PI-P1 (plateau of recovery from the primary lesion in the left $\mathrm{M} 1$ for the right hand) was compared with the performance at subsequent periods for the same hand following the secondary lesion in the right M1 (Ac-P2, Rec-P2 and PI-P2).

Modified Brinkman board task. The time course of the score for each hand in the modified Brinkman board task shows the effects of the primary and the secondary M1 lesions on the respective contralesional hand; right hand for primary left $\mathrm{M} 1$ lesion and left hand for secondary right M1 lesion (Fig. 2B). The scores reflected stable performance of the contralateral hands before each $\mathrm{M} 1$ lesion, after which the score dropped dramatically to zero for a few days, and then recovery took place 
during a period of increasing performance, up to a maximal and stable performance post-lesion (plateau). Cumulated scores and CTs data (Fig. 5) have been calculated for the time windows previously defined in Fig. 1, excluding the acute and recovery periods for the contralesional hand.

As expected, the primary and the secondary M1 lesions significantly decreased the scores and increased the CT of the contralesional hand during the post-lesion plateau periods (PI-P1 and PI-P2), as compared to prelesion (PreL) values of reference (Figs. $2 B$ and 5). After the primary lesion in the left $\mathrm{M} 1$ hand area, both the score and CT for the right hand were more strongly affected in the horizontal slots than in the vertical ones, as also reflected by the percentages of functional recovery (Table 1: Brinkman).

In the context of the role played by the intact M1 in the functional recovery from unilateral M1 lesion, the recovered performance of the right hand at PI-P1 was compared with the post-secondary lesion periods (Fig. 5A; white boxes). The score data demonstrate that the secondary lesion did not impair the recovered performance of the right hand (actually higher scores at all post-secondary lesion periods than at PI-P1), except a transient and modest decrease of the score for the horizontal wells, limited though to the Ac-P2 period and not statistically significant (Figs. 2B and 5A; Table 1). Very similar conclusions can be drawn from the CT data (Fig. 5A; Table 1), namely no significant increase of CT for the right hand following the secondary lesion (as compared to PI-P1), except a modest increase limited to the Ac-P2 period and not statistically significant. In summary, the modified Brinkman board task data (Fig. 5A white boxes) indicate that the intact M1 does not play a significant role in the recovery from the primary lesion at a post-lesion time point of 5 months.

Effects of the primary M1 lesion on the ipsilesional (left) hand were also observed, though clearly less strong than on the contralesional hand, as expected
(Figs. 2B and 5B; see also Table 1 for the percentages of performance with respect to the PreL of reference). Following the primary lesion in the left M1 hand area, the ipsilesional left hand (Fig. 5B) was transiently affected during the Ac-P1 period in the horizontal slots, especially the $\mathrm{CT}$, while the vertical slots were not affected, if not improved.

Modified Klüver board task: Grasping phase. The two sequential M1 lesions significantly increased the time intervals and modified the strategy to retrieve pellets from the four well sizes, for the contralesional hand during the post-lesion plateau periods (PI-P) (Fig. 6). The time intervals to grasp pellets in wells were much longer for the right hand after the primary lesion in the left M1 hand area (Fig. 6A), with percentages of incomplete recovery ranging from $43 \%$ to $61 \%$ across well diameters (Table 1; PI-P1 period, grasping time interval). Time intervals were also increased for the left hand after the secondary lesion in the right $M 1$ hand area (Fig. 6B), with percentages of recovery ranging from $60 \%$ to $87 \%$ (Table 1; PI-P2 period). As far as the contribution of the intact $\mathrm{M} 1$ in the recovered performance from the primary lesion is concerned, the pertinent grasping time interval data for the Klüver board task are shown in Fig. 6A (white boxes). The comparison of grasping time intervals at plateau PI-P1 and at periods subsequent to the secondary lesion shows a few modest increases at AC-P2 and/or Rec-P2 for the 15-mm and $21-\mathrm{mm}$ wells, but these differences were not statistically significant. For larger well diameters (30 and $40 \mathrm{~mm}$ ), the grasping time intervals were shorter after the secondary lesion than at PI-P1, consistent with an absence of detrimental effect of the secondary lesion on the recovery from the primary lesion for the right hand (Fig. 6A, white boxes).

The grasping strategy was analyzed by quantifying the different finger configurations used to retrieve the pellets from the four wells of different diameters (Fig. 6).

Table 1. Percentage of performance and/or functional recovery after the primary and the secondary M1 lesions, within the consecutive time windows (periods) as defined in Fig. 1, and for the motor parameters listed on the left. Data are presented for the 2 behavioral tasks (Brinkman and Klüver). The percentages were calculated with respect to the Pre-Lesion performance (PreL), before the primary lesion, at which the performance represents the $100 \%$ reference value (not listed in the table)

\begin{tabular}{|c|c|c|c|c|c|c|c|c|c|c|c|c|c|}
\hline & & \multicolumn{6}{|c|}{ Right hand } & \multicolumn{6}{|c|}{ Left hand } \\
\hline & & Ae-PI & Rec-P1 & Pl-P1 & Ac-P2 & Rec-P2 & PI-P2 & Ac-P1 & Rec-P1 & Pl-P1 & Ae-P2 & Rec-P2 & Pl-P2 \\
\hline \multirow{2}{*}{$\begin{array}{l}\text { Brinkman } \\
\text { score }\end{array}$} & Vertical & & & 69 & 72 & 77 & 80 & 96 & 111 & 101 & & & 89 \\
\hline & Horizontal & & & 45 & 37 & 45 & 53 & 88 & 97 & 100 & & & 86 \\
\hline \multirow{2}{*}{$\begin{array}{l}\text { Brinkman } \\
\text { CT }\end{array}$} & Vertical & & & 47 & 52 & 48 & 60 & 127 & 118 & 110 & & & 89 \\
\hline & Horizontal & & & 39 & 33 & 46 & 42 & 50 & 112 & 109 & & & 89 \\
\hline \multirow{4}{*}{$\begin{array}{l}\text { Klüver Board } \\
\text { grasping time } \\
\text { intervals }\end{array}$} & $15 \mathrm{~mm}$ & & & 61 & 57 & 52 & 69 & 89 & 91 & 91 & & & 60 \\
\hline & $21 \mathrm{~mm}$ & & & 55 & 67 & 62 & 75 & 113 & 114 & 110 & & & 77 \\
\hline & $30 \mathrm{~mm}$ & & & 54 & 58 & 63 & 69 & 103 & 107 & 101 & & & 84 \\
\hline & $40 \mathrm{~mm}$ & & & 43 & 49 & 50 & 52 & 108 & 105 & 110 & & & 87 \\
\hline \multirow{4}{*}{$\begin{array}{l}\text { Klüver Board } \\
\text { reaching time } \\
\text { intervals }\end{array}$} & $15 \mathrm{~mm}$ & & & 87 & 88 & 86 & 89 & 92 & 97 & 93 & & & 101 \\
\hline & $21 \mathrm{~mm}$ & & & 88 & 92 & 89 & 91 & 87 & 92 & 91 & & & 101 \\
\hline & $30 \mathrm{~mm}$ & & & 89 & 106 & 90 & 97 & 94 & 96 & 96 & & & 107 \\
\hline & $40 \mathrm{~mm}$ & & & 88 & 97 & 96 & 96 & 92 & 93 & 93 & & & 98 \\
\hline
\end{tabular}

For the Klüver board task, the diameter of the corresponding well is indicated in $\mathrm{mm}$. Data in bold for the right hand at period PI-P1 represents the stabilized performance after incomplete recovery from the primary lesion in the left M1 (less than 100\%) and for comparison with the data obtained in the next periods following the secondary lesion in the right M1. This comparison shows that the secondary lesion did not decrease the recovered performance of the right hand as the vast majority of \% values after the secondary lesion (at Ac-P2, Rec-P2 and PI-P2) are still higher than at PI-P1. 
The finger configuration indicates which fingers were used in addition to the thumb: for instance the index finger in addition to the thumb in the D2 configuration, the index finger and the middle finger in addition to the thumb in the D2-3 configuration, and so on. Invariably, for all pellet retrievals, D2 was inserted into the well to retrieve the pellet and the other fingers were progressively inserted from D3 to D5 when well diameters increased and post-lesion (Fig. 6). Representative examples of different finger configurations observed for the right hand pre-lesion (PreL) and at the plateau (PI-P1) after the primary lesion of left M1 are illustrated in the video sequences 1-4 (supplementary material, online version only). Notice that when the same finger configuration was used post-lesion, the retrieval time was longer than prelesion, corresponding to a residual deficit. With both hands, Mk-DG used progressively more fingers when grasping pellets from larger and larger well diameters in the PreL of reference (Fig. 6; period $1=$ Pre-L). More fingers were involved with the left hand in the two largest well diameters than with the right hand. Postprimary lesion (period $4=\mathrm{PI}-\mathrm{P} 1$ for the right hand), there was a switch in favor of finger configurations involving the combinations D2-D3 and D2-D3-D4. However, considering the effect of the secondary M1 lesion on the recovered performance of the right hand from the primary lesion in the left $\mathrm{M} 1$, the comparison has to be made between PI-P1 (period 4) and the AC$\mathrm{P} 2$, Rec-P2 and PI-P2 subsequent to the secondary lesion (periods $5-7$ in the bottom graph of Fig. 6). It appeared that the finger configurations are largely comparable during all these time windows for all well diameters, with only slightly more frequent use of more fingers. As for the time intervals (Fig. 6A), it can be concluded that the secondary lesion did not have a significant impact on the recovered grasping performance of the right hand from the primary lesion.

Effects on time intervals and grasping strategies (Fig. 6B) were also observed on the ipsilesional hand after the primary lesion in the left $\mathrm{M} 1$, but these effects were modest. For grasping time intervals, as compared to PreL, the effect was a small increase for the $15 \mathrm{~mm}$ well diameter and a decrease for the other 3 well diameters (see corresponding percentages of performance in Table 1; Left hand, periods PI-P1).

Modified Klüver board task: Reaching phase. The reaching phase of the movement was also affected following the two sequential M1 lesions, but to a lesser extent than the grasping phase. The primary lesion in the left M1 hand area significantly increased the time to reach the well for both hands, irrespective of the well diameters after the lever was released (Fig. 7A, B). The corresponding percentages of performance (timing intervals for reaching), as compared to pre-lesion, are indicated in Table 1 (time interval of reaching). Moreover, the right contralesional hand's fingers were over-extended, leading to a reduction of preshaping following the primary M1 lesion. Distances between index and thumb tips were larger than pre-lesion until reaching the well border (Fig. $7 \mathrm{~A}$ ), against which the index entered in contact first and then got pushed toward the thumb to further grasp the pellet. The indexthumb tips distances post-lesion however did not return to pre-lesion values and remained larger and stable during all subsequent periods following the primary lesion (Fig. 7A). There was no preshaping change of the ipsilesional (left) hand, such as the distance between the index and thumb tips, in relation to the primary lesion (see periods Ac-P1, Rec-P1 and PI-P1 in Fig. 7B).

Considering the secondary lesion in the right $\mathrm{M} 1$ hand area, the contralesional left hand exhibited post-lesion a transient modification of the preshaping properties, with an increase of the index-thumb tips' distance (Fig. 7B), mainly within the intermediate and terminal zones of the 2D movement trajectory. However, this increase of distance was limited to the recovery period (Rec-P2) and then returned to values of reference at plateau ( $\mathrm{Pl}-$ P2 in Fig. 7B).

As far as the role of the intact M1 in the functional recovery from the primary lesion in the left $M 1$ is concerned, the effect of the secondary lesion in the right M1 on the ipsilesional right hand was mainly a decrease of reaching time intervals as compared to PI-P1 (white boxes in Fig. 7A), in line with an absence of detrimental effect (slower reaching movement) of the secondary lesion on the recovered reaching performance. Similarly, the secondary lesion in the right M1 did not impact on the index-thumb tips distance (Fig. 7A, compare periods Ac-P2, Rec-P2 and PI-P2 with PI-P1). In summary, the secondary lesion did not modify the recovered reaching strategies from the primary lesion (Fig. 7A).

\section{Assessment of the lesions: MRI and histology}

Before performing the secondary M1 lesion (i.e. during the stable period of the post-lesion plateau PI-P1), the correct location of the primary lesion in the left M1 has been examined using MRI (as illustrated on one sagittal plane in Fig. 8A). The primary lesion was located in the expected left $\mathrm{M} 1$ and its volume was estimated at $21 \mathrm{~mm}^{3}$ (as derived from reconstruction of consecutive MRI planes) at a post-lesion time point corresponding to nearly 4 months.

Post-mortem, histological processing was performed in order to assess the volume and location of the two sequential $\mathrm{M} 1$ lesions at a time point corresponding to 2 months after the secondary lesion (Fig. 8B). The primary lesion, at the time of the secondary lesion plateau, extended from the surface down to the consecutive cortical layers and further into the subcortical white matter (Fig. 8B, left hemisphere). The primary cortical lesion had a volume of $32.2 \mathrm{~mm}^{3}$ in the gray matter and $1.9 \mathrm{~mm}^{3}$ subcortically in the white matter, adjacent to the gray matter lesion (Fig. 8C; left hemisphere). The secondary lesion exhibited a different anatomical characteristic, affecting more deeply the subcortical white matter (as seen in Nissl staining, Fig. $8 \mathrm{C}$ ), representing a volume of $3.4 \mathrm{~mm}^{3}$ whereas, in the gray matter, patchy zones of layer $\mathrm{V}$ without large pyramidal cells were observed in SMI-32 staining (Fig. 8B; right hemisphere). Although it was 


\section{A MRI image of primary lesion 4 months post- lesion}

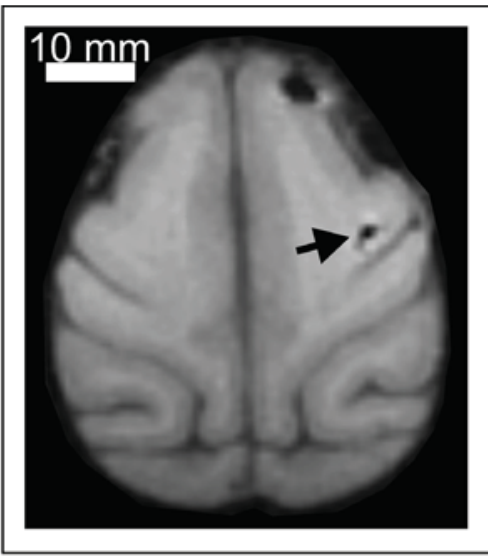

\section{B Left hemisphere Primary lesion with Ibotenic acid}
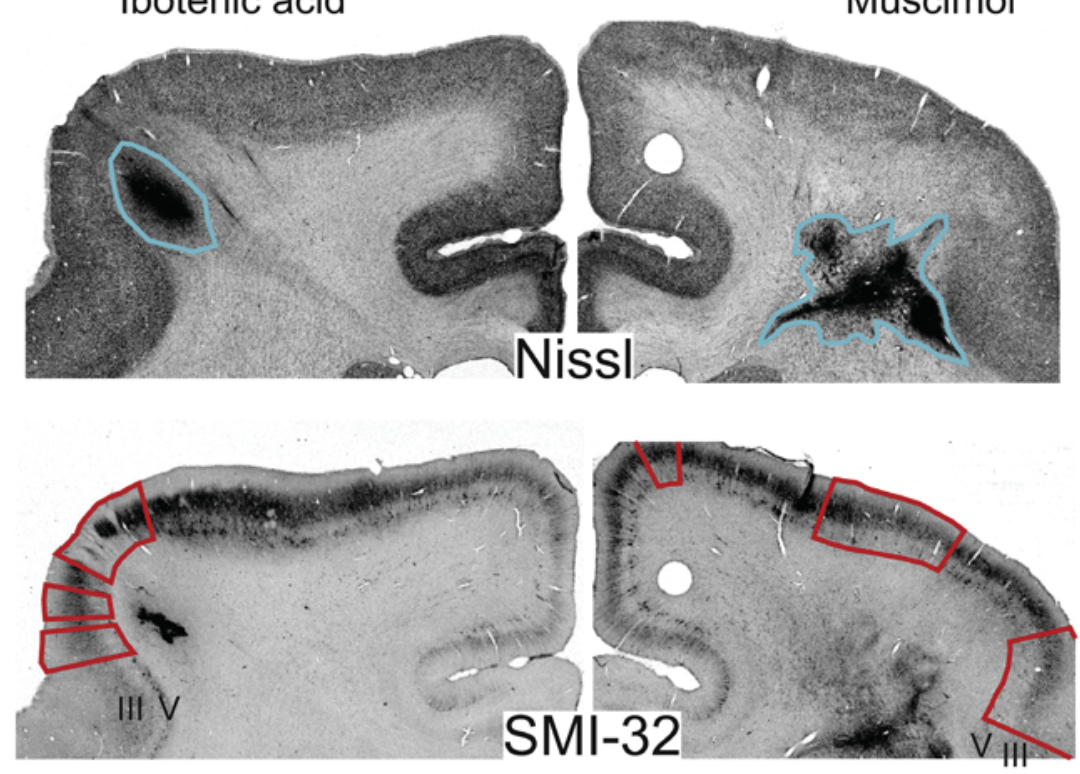

C

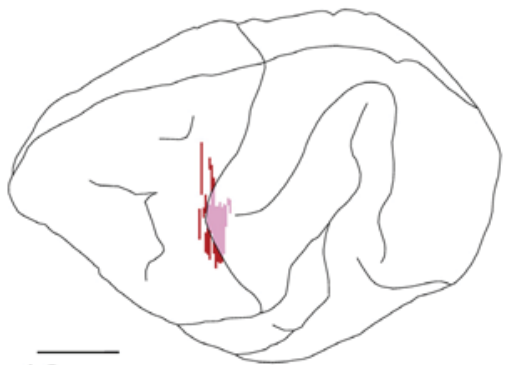

$10 \mathrm{~mm}$

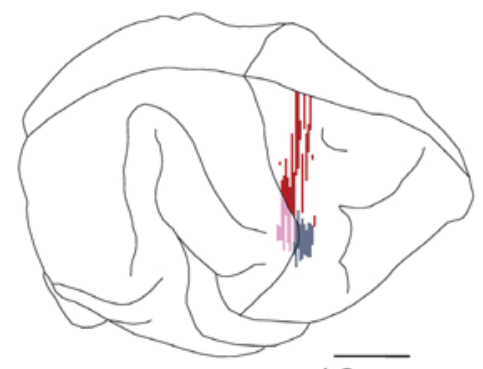

$\overline{10 \mathrm{~mm}}$

\section{$32.2 \mathrm{~mm}^{3} \quad$ SMI-32: Layer $\mathrm{V} \quad 37.5 \mathrm{~mm}^{3}$ \\ $1.9 \mathrm{~mm}^{3} \quad$ Nissl: Subcortical $\quad 3.4 \mathrm{~mm}^{3}$}

- Cortical lesion of the layer V = Cortical lesion of the layer V in the - Subcortical lesion in the white matter rostral bank of the central sulcus over-concentrated, it is unlikely that a small volume of muscimol targeting the gray matter generated such a lesion in the white matter. One may consider the possibility that the injection needle may have produced subsequent vascular damage, leading to the sub-cortical lesion. As a result, the wide loss of layer $V$ pyramidal neurons may result from retrograde degeneration following the white matter axonal injury, as the layer III neurons remained mostly intact, as observed on SMI-32 stained sections (Fig. 8B). These diffused and widely spread patches of layer $\mathrm{V}$ zones deprived of pyramidal neurons (as seen on SMI-32 stained sections) covered a relatively large volume of gray matter, corresponding to a lesion volume of $37.5 \mathrm{~mm}^{3}$, based on the layer $\mathrm{V}$ missing pyramidal neurons. The anatomical reconstructions show the exact location of the primary lesion in the left M1 hand area and the wider extent of the secondary

Fig. 8. MRI and anatomical representation of the M1 cortical lesions. (A) MRI acquisition (in the horizontal plane) of the brain of Mk-DG following the primary lesion of the left M1 hand area, showing the location of the lesion (arrow). (B) Nissl and SMI-32 staining of frontal histological sections of the two hemispheres of Mk-DG at low magnification. Nissl staining was used to delimit the subcortical lesion, delineated with the blue line and SMI32 staining to delimit the cortical lesion characterized by the missing layer $\mathrm{V}$, delineated with the red line. The hole on the right hemisphere next to the cingulate cortex is the cavity created by the insertion of a needle in the fixed brain in order to distinguish the right hemisphere from the left. (C) Lesion reconstructions on drawings of the corresponding left and right hemispheres (lack of layer V) on the surface (red area) and located in the rostral bank of the central sulcus (light red area) and subcortically (gray area). The subcortical lesion in the left hemisphere does not appear on this reconstruction, due to its overlap with the cortical lesion. This surface representation does not fully reflect the volume of the lesion indicated in $\mathrm{mm}^{3}$ below and derived from the serial section volume calculation, as explained in the method section. Actually, the hemisphere representation is not unfolded and, as a consequence, a lesion territory mostly in the rostral bank of the central sulcus (as on the left hemisphere) will yield a territory projection on the surface (red area) smaller than a similar lesion territory located mostly on the surface portion of M1 (as in the right hemisphere). For this reason, the quantified volume in $\mathrm{mm}^{3}$ is the correct estimate of the lesion extent. 
A

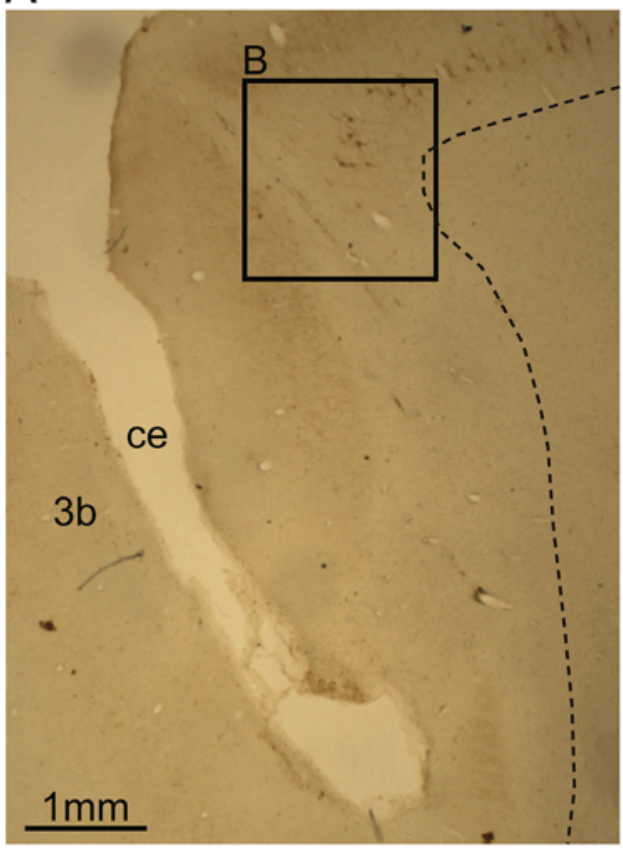

B

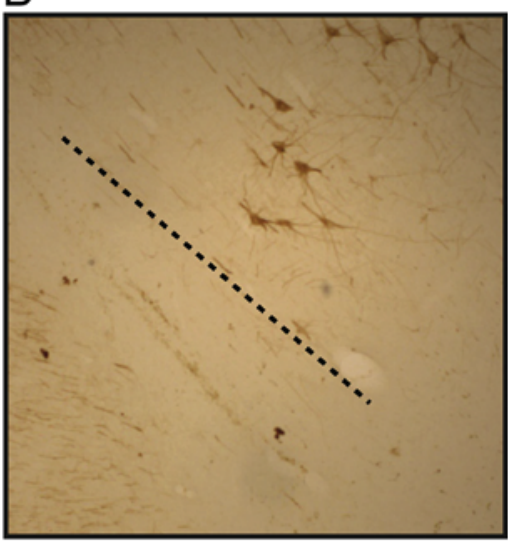

D

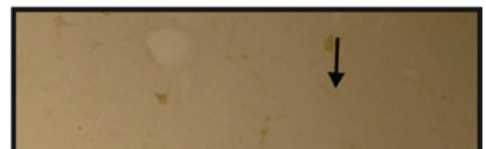

C

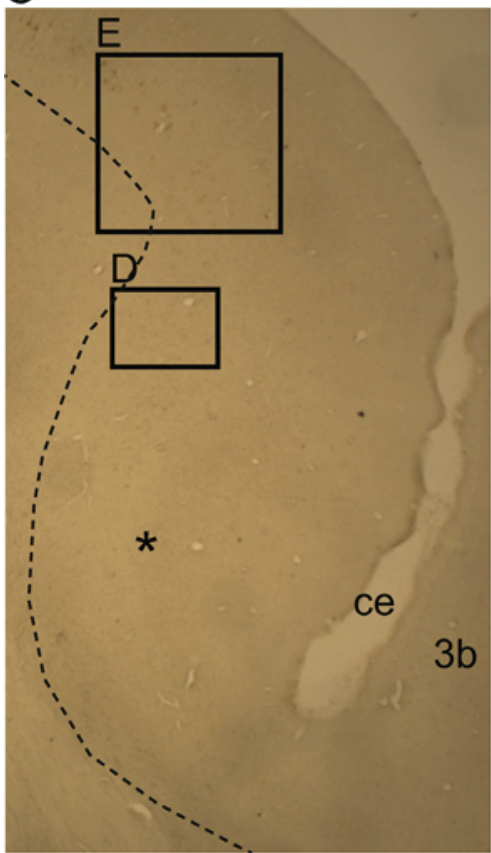

$E$
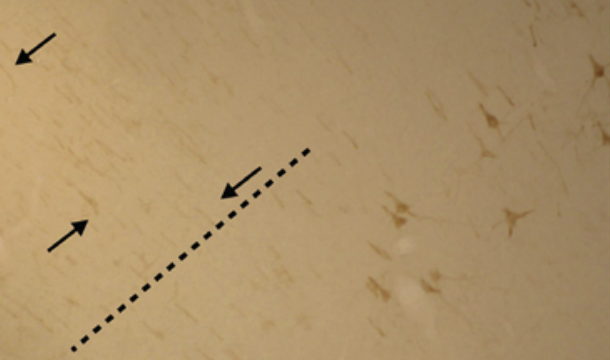

(1)

Fig. 9. Location of the lesions in M1 frontal sections of the brain of Mk-DG showing the rostral bank of the central sulcus in left M1 (panel A) and in right M1, corresponding to the "new M1" (Rathelot and Strick, 2009). Sections were processed to visualize the marker SMI-32, labeling mainly pyramidal neurons in layer V. On both sides, due to the lesion, there is a lack of SMI-32positive neurons along the rostral bank of the central sulcus (new M1) whereas the part of M1 on the surface of the hemisphere (old M1) is characterized by the presence of SMI-32-positive neurons, not affected by the lesion (see text for further description). The zones displayed by the rectangles $B, D$ and $E$ in panels $A$ and $C$ are shown at higher magnification on the right. The scale bar in panel A applies to panel $B$ as well. The higher magnifications panels $B, D$ and $E$ were enlarged by a factor of 3.2 as compared to panels $A$ and $C$. Central sulcus: ce; Somatosensory cortical area 3b: 3b; M1 hand area in rostral bank containing corticospinal neurons: *

lesion spreading medially beyond the right $\mathrm{M} 1$ hand area, toward the leg M1 area (Fig. 8C).
The hypothesis of the secondary M1 lesion occurring in several steps (muscimol effect first, vascular effect and finally retrograde degeneration of corticospinal neurons) is consistent with the behavioral observations during the few hours post-lesion, at least for the first two steps. As opposed to the ibotenic acid (primary) lesion on the opposite hemisphere (effect in $15 \mathrm{~min}$ after a $50 \mathrm{~min}$ infusion duration), the infusion of the overdosed muscimol injection provoked a deficit of the contralesional forelimb about $90 \mathrm{~min}$ after injection offset (flaccid paralysis; after a 10-min infusion duration). Five hours later, a more dramatic and more extensive deficit appeared, affecting nearly the entire left body hemi-side, from the lip to the leg (hyperextension). Twentyfour hours after injection of muscimol, the remaining deficit was mostly restricted to the left hand.

Referring to the partitioning of M1 (Rathelot and Strick, 2009) into a rostral part ("old M1") and a caudal part ("new M1"), the latter containing the corticomotoneuronal neurons of layer $V$ essential for skilled hand movements, it is of importance to assess whether the two lesions affected the mostly pertinent "new M1" territory, corresponding to the rostral bank of the central sulcus. Indeed, as illustrated in Fig. 9A, the primary lesion in the left hemisphere covered the entire rostral bank of the central sulcus (new M1), where all SMI-32 stained cells in layer $\mathrm{V}$ have disappeared. In contrast, in the part of M1 on the surface of the hemisphere (old M1), numerous SMI-32-positive neurons in layer $\mathrm{V}$ were still visible (Fig. 9, panels A and B). There was an abrupt transition between the lesioned territory and the mostly intact $M 1$, located where one expects the limit between new $\mathrm{M} 1$ and old M1 (dashed line in panel B of Fig. 9). The primary lesion in the left M1 thus impacted massively on the new M1 territory and only marginally on the old M1 (a few patchy zones with less SMI-32-positive neurons in layer $\mathrm{V}$ ). Along the rostrocaudal axis, the primary lesion covered a zone of $3.5 \mathrm{~mm}$, close to the expected extent of the hand area in the new M1. The secondary lesion in the right 
hemisphere also affected more the new M1 than the old $M 1$, although the latter was more affected than in the left hemisphere, with disrupted layer $\mathrm{V}$ zones medially (Fig. 8). In the new M1, as illustrated in Fig. 9 (panel C), the secondary lesion also generated a substantial loss of SMI-32-positive neurons along the rostral bank of the central sulcus, especially in the deep zone (asterisk in Fig. 9C). More superficially, still along the rostral bank of the central sulcus, there were few poorly stained SMI-32 neurons with a modified phenotype suggesting that, although surviving, they have been impacted by the overdosed muscimol (Fig. 9, panels $D$ and $E$ ). At the expected transition between the new M1 and the old M1 (dashed line in panel $E$ of Fig. 9), there was a progression from poorly stained and untypical layer V SMI-32 neurons in new M1 (on the right of the dashed line: black arrows) to better stained SMI-32 neurons and more typical neurons of layer $\mathrm{V}$ in the old M1 (left to the dashed line: open head arrows). Along the rostrocaudal axis, the secondary lesion covered an extent of $4.25 \mathrm{~mm}$, also roughly corresponding to the hand area size in the new M1.

\section{DISCUSSION}

This case report presents the effects on manual dexterity of two sequential permanent (long lasting) lesions consecutively targeting each $\mathrm{M} 1$ hand area, one hemisphere after the other. To summarize, the main findings are: (1) As expected, the primary cortical lesion in the left M1 hand area dramatically and irreversibly impaired the grasping ability of the contralesional right hand; the extent of these deleterious effects progressively increased from power grip, to precision grip and then to the more complex precision grip when associated with pro-supination movements and wrist deviations (horizontal wells in the modified Brinkman board task); (2) As expected, the secondary lesion in the right $\mathrm{M} 1$ hand area impaired the grasping ability of the contralesional left hand, indicating that the secondary lesion procedure was potent; (3) Overall, the secondary lesion, for the most part, did not have an impact on the recovered motor performance from the primary lesion; this observation suggests that, after a unilateral lesion of M1 hand area, the intact M1 does not play a major role in the functional recovery, at least at a time point of several months post-lesion; (4) The two behavioral tasks (modified Brinkman and Klüver boards) led to largely consistent conclusions regarding the effect of the secondary lesion, although they assess distinct but complementary motor parameters.

\section{Originality of the study}

To our knowledge this study, although limited to a case report, presents two original findings. Firstly, this is an original case of the same macaque monkey performing daily and in parallel the two main manual tasks currently reported in the literature, namely the modified Brinkman board task and the modified Klüver board task. This allows, to some extent, an original comparison of these two tasks. The "modified Brinkman board" task highlighted the important distinction between simple and complex precision grips (vertical versus horizontal wells, respectively). The complex precision grip has a higher vulnerability to $\mathrm{M} 1$ lesion, as it was much more impaired than the simple precision grip (Table 1: vertical versus horizontal slots). Such a distinction cannot be observed with the "modified Klüver board" task. The "modified Klüver board" task also highlighted large impairments of the power grip. Above all, it allowed for observations where the monkey was free to use or not a precision grip, clear modifications of grip strategies, which compensated well for impairments of precision grip. Thus, the change in strategy, toward the use of more power grips, explains the shorter time needed for grasping. Although precision grips were apparently used correctly after functional recovery, it appeared that the forced use of the precision grip in the smallest well diameter (excluding the use of digits 3-5) allowed the monkey to perform it with the help of the well border (index in contact first, then pushed toward the thumb); in contrast, in free conditions (larger well diameters), the power grip was clearly favored. Indeed, our results have highlighted an increased use of the D2-D3-D4 finger configuration for the right hand post-lesion instead of the D2-D3 finger configuration pre-lesion (Fig. 6). This reflects the switch of the grasping strategy after unilateral M1 lesion. Derived from the Klüver board task (with the hand placed on an initiation lever before each trial), the M1 lesions differently affected the distinct phases of the entire movement, namely the grasping phase and the reaching phase. Assessed distinctly with these two complementary tasks, our observations suggest that multiple aspects of the grip movement interact and contribute to compensate for grip impairment as a whole.

Secondly, the monkey Mk-DG is a nearly unique case reporting comprehensive quantitative data on the consequences of consecutive bilateral permanent M1 lesion in the hand area on one hemisphere and later on the other hemisphere, the secondary lesion occurring with enough delay to allow (incomplete) recovery from the primary lesion (at plateau). It could be argued that the secondary lesion was an incomplete gray matter damage in the intact M1 hand, thus influencing less the functional recovery than following the primary lesion. Nevertheless, the location of the secondary lesion in the white matter suggests that the effects were prominent. There is evidence that the deeper a lesion in the white matter the more extensive are the effects: functional recovery following lesion targeting upper limb has been shown to progressively decrease in parallel to a move to the lesion location from the cortex, corona radiate to internal capsule (Shelton and Reding, 2001; Morecraft et al., 2002, 2007). This allowed assessing the role of the contralateral intact M1 for long-term recovery from a unilateral M1 lesion. In earlier studies (Brown and Sherrington, 1913; Leyton and Sherrington, 1917; Ogden and Franz, 1917; Kennard, 1942), based on qualitative data derived from a sequential lesion bilaterally in the motor cortex, there was evidence that the secondary lesion of the intact motor cortex did not influence the recovery from the primary lesion. However, besides their 
qualitative dimension, the data were obtained from juvenile macaques (less than 2 years: Ogden and Franz, 1917; Kennard, 1942), an experimental condition different from the monkey included in the present study (10 years old). The chimpanzee data (Leyton and Sherrington, 1917) were most likely derived from adults (age not indicated though), but the sequential ablation experiment affecting the arm area bilaterally involved a primary lesion performed in two steps (60 days apart), a condition different from the primary lesion performed here in a single step. Furthermore, the time interval between the "last" primary lesion step and the secondary lesion on the other hemisphere was very short (1 month), insufficient to have reached a plateau of recovery. The other chimpanzee study (Brown and Sherrington, 1913) appears comparable to the present study for the sequential lesion schedule and reached qualitatively a conclusion fully in line with the present quantitative evidence that the intact M1 did not play a major role in the recovery from the primary lesion at a late time point.

As far as the recovery from the primary lesion is concerned, in the present study, observations performed in the housing facility at plateau also did not show visible remaining deficits for the same moving, climbing and eating behaviors as reported in the early studies mentioned above, suggesting complete recovery, in line with these early studies. The present study revealed however that the quantitative analysis of more challenging motor behaviors (modified Brinkman and Klüver board tasks) provided evidence for remaining deficits, corresponding to an incomplete functional recovery.

A limitation of the present study is that it is restricted to a single monkey, although in comparison to earlier qualitative studies of a sequential permanent lesion, this particular animal yielded a considerable amount of quantitative behavioral data, based on two different behavioral tests, leading to consistent conclusions. Moreover, the quantitative conclusions met here are fully in line with early qualitative observations, indicating that the present results, though limited to a single animal, can most likely be generalized to non-human primates.

\section{Muscimol-induced secondary lesion}

Muscimol was routinely used previously for reversible inactivation of cortical activity (e.g. Gallese et al., 1994; Kurata and Hoffman, 1994; Kermadi et al., 1997; Fogassi et al., 2001; Hoogewoud et al., 2013). How it generated a permanent lesion in the right M1 in the present study deserves some discussion, especially in relation to its accidental high dosage (5 times higher than for reversible inactivation). The possibility that muscimol at high dose played a direct role in generating a permanent lesion resides in the prospective toxicity of muscimol which, as GABAa agonist, generates chloride ion $\left(\mathrm{Cl}^{-}\right)$flux entry into the cell. Thus, the likelihood that a highly concentrated solution of muscimol could modify on the longterm the ionic flux leaving the GABAa channel in an open state causing ionic imbalance responsible for cell death is a subject of debate. The role of inhibitory neurotransmitter in neuronal death through lethal entry of $\mathrm{Cl}^{-}$has been suggested (Chen et al., 1998). Another study has proposed muscimol induction of neuronal death through GABAa auto-receptor (Honegger et al., 1998). Other authors have reported excitotoxic neuronal death through GABAa receptor, as the excitatory capacity expressed during development decreases the chloride gradient across the neuronal plasma membrane in case of long stimulation of the receptor (Xu et al., 2000). Nevertheless, as presented in the Results section (see also Fig. 8), the most likely scenario is a lesion occurring in a cascade of events, the infusion of muscimol followed by a vascular lesion in the white matter, leading then to a retrograde degeneration of layer $\mathrm{V}$ pyramidal neurons in a large portion of M1. Importantly, the secondary lesion induced substantial and permanent damage, visible on histological sections, which is not the case after muscimol infusions used for reversible cortical inactivation (as observed in the study of Hoogewoud et al., 2013). This secondary lesion in the right M1 generated a clear behavioral deficit in the contralesional left forelimb, suggesting that it was a potent lesion. Consequently, this secondary lesion was pertinent to address the main aim of the present study, mainly assess the role of the intact $\mathrm{M} 1$ on the recovery from the primary unilateral M1 lesion (in the left M1) at plateau.

\section{Behavioral results}

The two behavioral tasks used in the present report generated largely consistent and complementary results with respect to the main conclusions of the present study, especially in relation to the absence of major effect of the secondary lesion on the functional recovery from the primary M1 lesion. An exception is the discrepancy noted between the two mostly comparable CTs derived from the vertical slots in the modified Brinkman board task and the grasping times collected from the smallest well diameter in the modified Klüver board task. More precisely, in the vertical wells of the modified Brinkman board task, there was a complete recovery at $\mathrm{PI}-\mathrm{P} 2$ (Fig. 5A, CTs), whereas a grasping time interval deficit remained at PI-P2 for the $15 \mathrm{~mm}$ well of the Klüver board task (Fig. 6A). Multiple hypotheses can be proposed, but two of them may have an equal or cumulative impact. The first explanation proposes that the high number of trials performed in the modified Klüver board task has increased the probability that subtle changes may still be statistically significant. The second explanation is consistent with the negative effect associated with the increase of well depth on the recovery of precision grip following injury (Fukushima et al., 2007), as the wells of the modified Klüver board are deeper than the wells of the modified Brinkman board.

A limitation of the present study is that the behavioral consequences of the primary and secondary lesions cannot be easily compared, as the two appear different from each other. The primary lesion affected all layers of the gray matter while the secondary lesion affected mostly the layer V, largely sparing layer III, thus possibly preserving some corticocortical projections originating from superficial cortical layer of $M 1$ to 
premotor areas (PMv and PMd), to the supplementary motor area (SMA) and to the cingulate motor areas (CMA) (Leichnetz, 1986; Luppino and Rizzolatti, 2000; Dum and Strick, 2005), as well as local circuits. This is of major interest considering the proposed role of the secondary motor areas for functional recovery following M1 lesion, especially the role of the premotor cortex (Liu and Rouiller, 1999; Hoogewoud et al., 2013; Murata et al., 2015). Thus, the spared layer III after the secondary lesion partially preserved the highly interconnected neural circuits between M1, PMv and PMd, possibly allowing better functional recovery following $\mathrm{M} 1$ lesion, as compared to a cortical lesion affecting all cortical layers.

\section{Role of the ipsilateral M1 in complex hand movements}

Our results suggest an ipsilateral and/or a bilateral role of the M1 hand area in the control of voluntary movements. In line with a previous report from our laboratory (Bashir et al., 2012), the present study shows that the lesion of the $\mathrm{M} 1$ hand area has a limited transient deleterious effect on the ipsilesional hand, though limited to complex precision grip synergies. Similarly, the secondary lesion also transiently affected the precision grip of the ipsilesional hand. These data are consistent with the notion that bilateral M1 activation is present in the case of complex finger movements (Shibasaki et al., 1993; Salmelin et al., 1995; Chen et al., 1997; Ehrsson et al., 2000). Moreover, deficits of wrist movement ipsilateral to a stroke were reported in hemiparetic subjects (Yarosh et al., 2004). Therefore, the present results sustain the role, though not crucial, of an ipsilateral and/or a bilateral M1 implication for complex movements, thus affected by a unilateral lesion, however rapidly compensated and recovered.

\section{Role of the intact M1 in the spontaneous functional recovery}

In accordance with a previous report on rat (Shanina et al., 2006), the secondary M1 permanent lesion on the right hemisphere did not abolish the functional recovery occurring after the primary M1 permanent lesion on the left hemisphere, indicating that, following unilateral M1 lesion limited to the hand area, the intact hemisphere does not play a crucial role in the long-term functional recovery of the contralesional hand. This observation is consistent with the reported absence of effect of a reversible secondary lesion of the intact M1 on the recovery of manual dexterity from a unilateral M1 permanent lesion on the contralesional affected hand (Liu and Rouiller, 1999). The present confirmation of the absence of a role played by the intact M1 based on permanent (longlasting) lesion is of importance as the previous observation was derived from reversible inactivation (Liu and Rouiller, 1999) using a low dose of muscimol, which may not have fully inactivated the intact M1. Moreover, reversible inactivation with a low dose of muscimol does not allow for the observation of the effects of long-term inactivation of intact M1. In both studies (Liu and Rouiller, 1999; present study), the possibility that a contribution of the intact M1 in the functional recovery after unilateral M1 lesion was not observed may be due to the moderate size of the primary $\mathrm{M} 1$ lesion, leaving open the possibility that recovery from a larger lesion may possibly involve intact $M 1$, as suggested in previous reports (Liu and Rouiller, 1999; Biernaskie et al., 2005) and supported by a recent tract-tracing study in monkeys subjected to large unilateral cortical lesion (Morecraft et al., 2016).

\section{Role of M1 in the early and late periods of recovery}

Our results showing a delayed onset of the functional recovery following the secondary lesion as compared to the time course of functional recovery observed following the primary lesion for the different parameters assessed with the behavioral motor tasks (Fig. 4) are in agreement with a role of M1 in early stages of recovery. The present conclusion that intact M1 does not play a role in the functional recovery from a unilateral lesion of M1 applies for a time point at 5 months post-primary lesion. Another issue is the role of intact M1 during the early period of recovery following unilateral M1 injury, specifically for complex fine manual dexterity. A recent review (Dancause et al., 2015) listed reports emphasizing the beneficial effects of contralesional cortex inhibition on functional recovery following unilateral stroke when applied rapidly and for prolonged duration; in contrast when the inhibition of the contralesional cortex was delayed or applied for shorter durations such beneficial effects were lost. These considerations may not concern the effects of the secondary M1 lesion on the primarily affected hand functional recovery in the present study, the secondary M1 lesion taking place long after the primary M1 lesion. There is evidence that the normally masked ipsilateral connections of the intact contralesional cortex were revealed by a unilateral M1 cortical lesion in humans (Netz et al., 1997). In the present study, the different time courses of recovery observed following the two M1 lesions could be related to the absence of these connections at the time of the secondary M1 lesion. Furthermore, an increase of contralesional cortex activity was reported at an early stage following unilateral motor cortex lesion, then followed by an extension of the increased cortical activity to non-primary motor areas (Rehme et al., 2011). All together, these data are in agreement with the suggested role played by the contralesional M1 in the early stage of the recovery, before occurrence of subsequent plasticity. Consequently, the primary lesioned M1 was no longer able to support the early stage of the recovery following the secondary lesion of the intact hemisphere, explaining the observed delayed onset of the recovery of higher motor functions, before being taken over at a later stage, by non-primary motor areas.

The role of the interhemispheric disinhibition has been suggested to explain the observed motor improvement of the ipsilesional hand following the M1 lesion (Figs. 5B and 6B; percentages above 100\% in Table 1 for the left hand in Ac-P1, Rec-P1 and PI-P1 periods; see also Darling et al., 2011a; Manganotti et al., 2008; Kaeser et al., 2010). Using transcranial magnetic stimulation, the unaffected hemisphere has been reported to be disinhibited during the early period following a unilateral 
stroke, as suggested by the loss of the interhemispheric connection originating from the lesioned hemisphere (Shimizu et al., 2002). However, the increase of the fMRI activity observed early following a stroke in the unaffected hemisphere was reduced in relation to behavioral recovery in stroke patient (Ward et al., 2003). Thus, the interhemispheric disinhibition did not appear to support a role of M1 in these unaffected hand improvements at long-term and thus appeared more likely to play a role in the early period following the cortical lesion.

Further studies are needed to elucidate the mechanisms sustaining the functional recovery, probably not implying a direct role of intact contralateral M1. A plastic reorganization of neural circuits, involving non-primary motor areas, has been shown to take place (Dancause et al., 2005; McNeal et al., 2010; Hamadjida et al., 2012). There is recent evidence for a cortical somatosensory influence on the mechanisms of functional recovery after frontal lobe lesion including $\mathrm{M} 1$ (Morecraft et al., 2015, 2016).

\section{CONCLUSION}

This original, quantitative case report of two subsequent M1 permanent lesions offers some insight into the role of $\mathrm{M} 1$ in and execution of normal movements and in the functional recovery from unilateral permanent lesion of the $\mathrm{M} 1$ hand area. The results can be summarized as follows: (1) after a unilateral lesion of the M1 hand area, the hypothesis that the intact contralateral M1 does not play a major role in the long-term functional recovery was verified; (2) a bilateral, but not crucial, role of M1 in the execution of complex manual dexterity tasks was observed.

\section{AUTHORS CONTRIBUTIONS}

Julie Savidan participated in the experimental design, implemented the modified Klüver board device, trained the animal, participated in the surgeries and lesions, collected the data, analyzed the data and wrote the manuscript. Mélanie Kaeser participated in animal training and surgery. Abderraouf Belhaj-Saïf participated in the experimental design, contributed to both the development of the modified Klüver board device, and to the animal training. Eric Schmidlin participated in the experimental design (including ICMS and MRI acquisition), performed the surgery and lesions. Eric M. Rouiller supervised and contributed to the experimental design, the modified Klüver board implementation, the surgery and lesions, data analyses and wrote the manuscript.

\section{CONFLICT OF INTEREST}

None.

\section{GRANT SPONSORS}

Swiss National Science Foundation, Grants No 3161857.00, 310000-110005, 31003A-132465, 310030B149643 (EMR), the National Centre of Competence in
Research (NCCR) on "Neural plasticity and repair"; Novartis Foundation; The Christopher Reeves Foundation (Springfield, NJ, USA); The Swiss Primate Competence Centre for Research (SPCCR: http://www. unifr.ch/neuro/rouiller/SPCCR/welcome.html).

Acknowledgments-The authors wish to thank the technical assistance of Véronique Moret, Christine Roulin, and Christiane Marti (histology), Laurent Bossy and Jacques Maillard (animal care taking), André Gaillard (mechanics), Bernard Aebischer and Andrea Francovich (electronics), Laurent Monney (informatics). We thank Dr. Michael Harvey and Dr. Karen Bunday for editing the final version of the manuscript.

\section{REFERENCES}

Babiloni C, Carducci F, Pizzella V, Indovina I, Romani GL, Rossini PM, Babiloni F (1999) Bilateral neuromagnetic activation of human primary sensorimotor cortex in preparation and execution of unilateral voluntary finger movements. Brain Res 827:234-236.

Bashir S, Kaeser M, Wyss A, Hamadjida A, Liu Y, Bloch J, Brunet JF, Belhaj-Saif A, Rouiller EM (2012) Short-term effects of unilateral lesion of the primary motor cortex (M1) on ipsilesional hand dexterity in adult macaque monkeys. Brain Struct Funct 217:63-79.

Beaud ML, Schmidlin E, Wannier T, Freund P, Bloch J, Mir A, Schwab ME, Rouiller EM (2008) Anti-Nogo-A antibody treatment does not prevent cell body shrinkage in the motor cortex in adult monkeys subjected to unilateral cervical cord lesion. BMC Neurosci 9:5.

Beaud ML, Rouiller EM, Bloch J, Mir A, Schwab ME, Wannier T, Schmidlin E (2012) Invasion of lesion territory by regenerating fibers after spinal cord injury in adult macaque monkeys. Neurosci 227:271-282.

Biernaskie J, Szymanska A, Windle V, Corbett D (2005) Bihemispheric contribution to functional motor recovery of the affected forelimb following focal ischemic brain injury in rats. Eur $\mathrm{J}$ Neurosci 21:989-999.

Brinkman J, Kuypers HGJM (1973) Cerebral control of contralateral and ipsilateral arm, hand and finger movements in the split-brain rhesus monkey. Brain 96:653-674.

Brinkman C (1984) Supplementary motor area of the monkey's cerebral cortex: short- and long-term deficits after unilateral ablation and the effects of subsequent callosal section. J Neurosci 4:918-929.

Brochier T, Boudreau MJ, Pare M, Smith AM (1999) The effects of muscimol inactivation of small regions of motor and somatosensory cortex on independent finger movements and force control in the precision grip. Exp Brain Res 128:31-40.

Brown GT, Sherrington CS (1913) Note on the functions of the cortex cerebri. J. Physiol. (London) 46(suppl):xxii.

Brown AR, Teskey GC (2014) Motor cortex is functionally organized as a set of spatially distinct representations for complex movements. J Neurosci 34:13574-13585.

Chatagny P, Badoud S, Kaeser M, Gindrat AD, Savidan J, Fregosi M, Moret V, Roulin C, Schmidlin E, Rouiller EM (2013) Distinction between hand dominance and hand preference in primates: a behavioral investigation of manual dexterity in nonhuman primates (macaques) and human subjects. Brain Behav 3:575-595.

Chen R, Gerloff C, Hallett M, Cohen LG (1997) Involvement of the ipsilateral motor cortex in finger movements of different complexities. Ann Neurol 41:247-254.

Chen Q, Olney JW, Lukasiewicz PD, Almli T, Romano C (1998) Ca2 + -independent excitotoxic neurodegeneration in isolated retina, an intact neural net: a role for $\mathrm{Cl}$ - and inhibitory transmitters. Mol Pharmacol 53:564-572. 
Cirstea MC, Levin MF (2000) Compensatory strategies for reaching in stroke. Brain 123(Pt 5):940-953.

Dancause N, Barbay S, Frost SB, Plautz EJ, Chen D, Zoubina EV Stowe AM, Nudo RJ (2005) Extensive cortical rewiring after brain injury. J Neurosci 25:10167-10179.

Dancause N, Touvykine B, Mansoori BK (2015) Inhibition of the contralesional hemisphere after stroke: reviewing a few of the building blocks with a focus on animal models. Prog Brain Res 218:361-387.

Dancause N (2006) Vicarious function of remote cortex following stroke: recent evidence from human and animal studies. Neuroscientist 12:489-499.

Darling WG, Pizzimenti MA, Rotella DL, Peterson CR, Hynes SM, Ge J, Solon K, McNeal DW, Stilwell-Morecraft KS, Morecraft RJ (2009) Volumetric effects of motor cortex injury on recovery of dexterous movements. Exp Neurol 220:90-108.

Darling WG, Pizzimenti MA, Hynes SM, Rotella DL, Headley G, Ge J, Stilwell-Morecraft KS, McNeal DW, Solon-Cline KM, Morecraft RJ (2011a) Volumetric effects of motor cortex injury on recovery of ipsilesional dexterous movements. Exp Neurol 231:56-71.

Darling WG, Pizzimenti MA, Morecraft RJ (2011b) Functional recovery following motor cortex lesions in non-human primates: experimental implications for human stroke patients. J Integr Neurosci 10:353-384.

Darling WG, Helle N, Pizzimenti MA, Rotella DL, Hynes SM, Ge J, Stilwell-Morecraft KS, Morecraft RJ (2013) Laterality affects spontaneous recovery of contralateral hand motor function following motor cortex injury in rhesus monkeys. Exp Brain Res 228:9-24.

Darling WG, Morecraft RJ, Rotella DL, Pizzimenti MA, Ge J, StilwellMorecraft KS, Zhang H, Soliman H, Seecharan D, Edwards I, McNeal D, Nudo RJ, Cheney P (2014) Recovery of precision grasping after motor cortex lesion does not require forced use of the impaired hand in Macaca mulatta. Exp Brain Res 232:3929-3938.

Dum RP, Strick PL (2005) Frontal lobe inputs to the digit representations of the motor areas on the lateral surface of the hemisphere. J Neurosci 25:1375-1386.

Ehrsson HH, Fagergren A, Jonsson T, Westling G, Johansson RS Forssberg H (2000) Cortical activity in precision- versus powergrip tasks: an fMRI study. J Neurophysiol 83:528-536.

Fogassi L, Gallese V, Buccino G, Craighero L, Fadiga L, Rizzolatti G (2001) Cortical mechanism for the visual guidance of hand grasping movements in the monkey: A reversible inactivation study. Brain 124:571-586.

Freund P, Schmidlin E, Wannier T, Bloch J, Mir A, Schwab ME, Rouiller EM (2006) Nogo-A-specific antibody treatment enhances sprouting and functional recovery after cervical lesion in adult primates. Nature Med 12:790-792.

Freund P, Wannier T, Schmidlin E, Bloch J, Mir A, Schwab ME Rouiller EM (2007) Anti-Nogo-A antibody treatment enhances sprouting of corticospinal axons rostral to a unilateral cervical spinal cord lesion in adult macaque monkey. J Comp Neurol 502:644-659.

Freund P, Schmidlin E, Wannier T, Bloch J, Mir A, Schwab ME, Rouiller EM (2009) Anti-Nogo-A antibody treatment promotes recovery of manual dexterity after unilateral cervical lesion in adult primates-re-examination and extension of behavioral data. Eur J Neurosci 29:983-996.

Fukushima J, Kasahara S, Asaka T, Saito H, Yamanaka M (2007) Behavioral findings during recovery after experimental stroke in monkeys-Assessment with modified hand performance test. J Phys Ther Sci 19:33-40.

Gallese V, Murata A, Kaseda M, Niki N, Sakata H (1994) Deficit of hand preshaping after muscimol injection in monkey parietal cortex. NeuroReport 5:1525-1529.

Glees P, Cole J (1950) Recovery of skilled motor functions after small repeated lesions of motor cortex in macaque. J Neurophysiol 13:137-148

Gonzalez CLR, Gharbawie OA, Williams PT, Kleim JA, Kolb B, Whishaw IQ (2004) Evidence for bilateral control of skilled movements: ipsilateral skilled forelimb reaching deficits and functional recovery in rats follow motor cortex and lateral frontal cortex lesions. Eur J Neurosci 20:3442-3452.

Hamadjida A, Wyss AF, Mir A, Schwab ME, Belhaj-Saif A, Rouiller EM (2012) Influence of anti-Nogo-A antibody treatment on the reorganization of callosal connectivity of the premotor cortical areas following unilateral lesion of primary motor cortex (M1) in adult macaque monkeys. Exp Brain Res 223:321-340.

Hoffman DS, Strick PL (1995) Effects of a primary motor cortex lesion on step-tracking movements of the wrist. $J$ Neurophysiol 73:891-895.

Honegger P, Pardo B, Monnet-Tschudi F (1998) Muscimol-induced death of GABAergic neurons in rat brain aggregating cell cultures. Brain Res Dev Brain Res 105:219-225.

Hoogewoud F, Hamadjida A, Wyss AF, Mir A, Schwab ME, BelhajSaif A, Rouiller EM (2013) Comparison of functional recovery of manual dexterity after unilateral spinal cord lesion or motor cortex lesion in adult macaque monkeys. Front Neurol 4:101.

Jaillard A, Martin CD, Garambois K, Lebas JF, Hommel M (2005) Vicarious function within the human primary motor cortex? A longitudinal fMRI stroke study. Brain 128:1122-1138.

Kaeser M, Wyss AF, Bashir S, Hamadjida A, Liu Y, Bloch J, Brunet JF, Belhaj-Saif A, Rouiller EM (2010) Effects of unilateral motor cortex lesion on ipsilesional hand's reach and grasp performance in monkeys: relationship with recovery in the contralesional hand. J Neurophysiol 103:1630-1645.

Kaeser M, Brunet JF, Wyss A, Belhaj-Saif A, Liu Y, Hamadjida A Rouiller EM, Bloch J (2011) Autologous adult cortical cell transplantation enhances functional recovery following unilateral lesion of motor cortex in primates: a pilot study. Neurosurgery 68:1405-1417.

Kaeser M, Wannier T, Brunet JF, Wyss A, Bloch J, Rouiller EM (2013) Representation of motor habit in a sequence of repetitive reach and grasp movements performed by macaque monkeys: evidence for a contribution of the dorsolateral prefrontal cortex. Cortex 49:1404-1419.

Kaeser M, Chatagny P, Gindrat AD, Savidan J, Badoud S, Fregosi M, Moret V, Roulin C, Schmidlin E, Rouiller E (2014) Variability of manual dexterity performance in non-human primates (macaca fascicularis). Int J Comp Psychol 27:295-325.

Kennard MA (1942) Cortical reorganization of motor function: studies on series of monkeys of various ages from infancy to maturity. Arch Neurol Psychiatr 48:227-240.

Kermadi I, Liu Y, Tempini A, Rouiller EM (1997) Effects of reversible inactivation of the supplementary motor area (SMA) on unimanual grasp and bimanual pull and grasp performance in monkeys. Somatosens Mot Res 14:268-280.

Kurata K, Hoffman DS (1994) Differential effects of muscimol microinjection into dorsal and ventral aspects of the premotor cortex of monkeys. J Neurophysiol 71:1151-1164.

Lanz F, Lanz X, Scherly A, Moret V, Gaillard A, Gruner P, Hoogewoud HM, Belhaj-Saif A, Loquet G, Rouiller EM (2013) Refined methodology for implantation of a head fixation device and chronic recording chambers in non-human primates. J Neurosci Methods 219:262-270.

Leichnetz GR (1986) Afferent and efferent connections of the dorsolateral precentral gyrus (area 4, hand/arm region) in the macaque monkey, with comparisons to area 8. J Comp Neurol 254:460-492.

Leyton ASF, Sherrington CS (1917) Observations on the excitable cortex of the chimpanzee, orang-utan, and gorilla. Exp Physiol 11:135-221.

Liu Y, Rouiller EM (1999) Mechanisms of recovery of dexterity following unilateral lesion of the sensorimotor cortex in adult monkeys. Exp Brain Res 128:149-159.

Luppino G, Rizzolatti G (2000) The organization of the frontal motor cortex. News Phys Sci 15:219-224.

Manganotti P, Acler M, Zanette GP, Smania N, Fiaschi A (2008) Motor cortical disinhibition during early and late recovery after stroke. Neurorehabil Neural Repair 22:396-403. 
Martin JH (1991) Autoradiographic estimation of the extent of reversible inactivation produced by microinjection of lidocaine and muscimol in the rat. Neurosci Lett 127:160-164.

McNeal DW, Darling WG, Ge J, Stilwell-Morecraft KS, Solon KM, Hynes SM, Pizzimenti MA, Rotella DL, Vanadurongvan T, Morecraft RJ (2010) Selective long-term reorganization of the corticospinal projection from the supplementary motor cortex following recovery from lateral motor cortex injury. J Comp Neurol 518:586-621.

Milliken GW, Plautz EJ, Nudo RJ (2013) Distal forelimb representations in primary motor cortex are redistributed after forelimb restriction: a longitudinal study in adult squirrel monkeys. J Neurophysiol 109:1268-1282.

Morecraft RJ, Herrick JL, Stilwell-Morecraft KS, Louie JL, Schroeder CM, Ottenbacher JG, Schoolfield MW (2002) Localization of arm representation in the corona radiata and internal capsule in the non-human primate. Brain 125:176-198.

Morecraft RJ, McNeal DW, Stilwell-Morecraft KS, Dvanajscak Z, Ge $J$, Schneider P (2007) Localization of arm representation in the cerebral peduncle of the non-human primate. J Comp Neurol 504:149-167.

Morecraft RJ, Ge J, Stilwell-Morecraft KS, McNeal DW, Hynes SM Pizzimenti MA, Rotella DL, Darling WG (2015) Vulnerability of the medial frontal corticospinal projection accompanies combined lateral frontal and parietal cortex injury in rhesus monkey. J Comp Neurol 523:669-697.

Morecraft RJ, Ge J, Stilwell-Morecraft KS, McNeal DW, Hynes SM, Pizzimenti MA, Rotella DL, Darling WG (2016) Frontal and frontoparietal injury differentially affect the ipsilateral corticospinal projection from the nonlesioned hemisphere in monkey (Macaca mulatta). J Comp Neurol 524:380-407.

Murata Y, Higo N, Oishi T, Yamashita A, Matsuda K, Hayashi M, Yamane S (2008) Effects of motor training on the recovery of manual dexterity after primary motor cortex lesion in macaque monkeys. J Neurophysiol 99:773-786.

Murata Y, Higo N, Hayashi T, Nishimura Y, Sugiyama Y, Oishi T, Tsukada H, Isa T, Onoe H (2015) Temporal plasticity involved in recovery from manual dexterity deficit after motor cortex lesion in macaque monkeys. J Neurosci 35:84-95.

Netz J, Lammers T, Homberg V (1997) Reorganization of motor output in the non-affected hemisphere after stroke. Brain 120(Pt 9):1579-1586.

Nudo RJ, Barbay S (2014) The mechanisms and neurophysiology of recovery from stroke. Stroke Recovery Rehab.

Nudo RJ, Jenkins WM, Merzenich MM, Prejean T, Grenda R (1992) Neurophysiological correlates of hand preference in primary motor cortex of adult squirrel monkeys. J Neurosci 12:2918-2947.

Nudo RJ (2006) Mechanisms for recovery of motor function following cortical damage. Curr Opin Neurobiol 16:638-644.

Ogden R, Franz SI (1917) On cerebral motor control: the recovery from experimentally produced hemiplegia. Psychobiology $1: 33-47$

Olivier E, Davare M, Andres M, Fadiga L (2007) Precision grasping in humans: from motor control to cognition. Curr Opin Neurobiol $17: 644-648$

Peuser J, Belhaj-Saif A, Hamadjida A, Schmidlin E, Gindrat AD, Volker AC, Zakharov P, Hoogewoud HM, Rouiller EM, Scheffold F (2011) Follow-up of cortical activity and structure after lesion with laser speckle imaging and magnetic resonance imaging in nonhuman primates. J Biomed Opt 16:096011.

Pizzimenti MA, Darling WG, Rotella DL, McNeal DW, Herrick JL, Ge J, Stilwell-Morecraft KS, Morecraft RJ (2007) Measurement of reaching kinematics and prehensile dexterity in nonhuman primates. J Neurophysiol 98:1015-1029.

Qi HX, Gharbawie OA, Wynne KW, Kaas JH (2013) Impairment and recovery of hand use after unilateral section of the dorsal columns of the spinal cord in squirrel monkeys. Behav Brain Res 252:363-376.

Rathelot JA, Strick PP (2009) Subdivisions of primary motor cortex based on cortico-motoneuronal cells. PNAS 106:918-923.
Rehme AK, Fink GR, Von Cramon DY, Grefkes C (2011) The role of the contralesional motor cortex for motor recovery in the early days after stroke assessed with longitudinal FMRI. Cereb. Cortex 21:756-768.

Rizzolatti G, Luppino G (2001) The cortical motor system. Neuron 31:889-901.

Salmelin R, Forss N, Knuutila J, Hari R (1995) Bilateral activation of the human somatomotor cortex by distal hand movements. EEG Clin Neurophysiol 95:444-452.

Schieber MH, Poliakov AV (1998) Partial inactivation of the primary motor cortex hand area: effects on individuated finger movements. J Neurosci 18:9038-9054.

Schmidlin E, Wannier T, Bloch J, Rouiller EM (2004) Progressive plastic changes in the hand representation of the primary motor cortex parallel incomplete recovery from a unilateral section of the corticospinal tract at cervical level in monkeys. Brain Res 1017:172-183.

Schmidlin E, Wannier T, Bloch J, Belhaj-Saïf A, Wyss A, Rouiller EM (2005) Reduction of the hand representation in the ipsilateral primary motor cortex following unilateral section of the corticospinal tract at cervical level in monkeys. BMC Neurosci 6:56.

Schmidlin E, Brochier T, Maier MA, Kirkwood PA, Lemon RN (2008) Pronounced reduction of digit motor responses evoked from macaque ventral premotor cortex after reversible inactivation of the primary motor cortex hand area. J Neurosci 28:5772-5783.

Schmidlin E, Kaeser M, Gindrat AD, Savidan J, Chatagny P, Badoud S, Hamadjida A, Beaud ML, Wannier T, Belhaj-Saif A, Rouiller EM (2011) Behavioral assessment of manual dexterity in non-human primates. J Vis Exp:3258.

Shanina EV, Schallert T, Witte OW, Redecker C (2006) Behavioral recovery from unilateral photothrombotic infarcts of the forelimb sensorimotor cortex in rats: role of the contralateral cortex. Neurosci 139:1495-1506.

Shelton FN, Reding MJ (2001) Effect of lesion location on upper limb motor recovery after stroke. Stroke 32:107-112.

Shibasaki H, Sadato N, Lyshkow H, Yonekura Y, Honda M, Nagamine T, Suwazono S, Magata Y, Ikeda A, Miyazaki M (1993) Both primary motor cortex and supplementary motor area play an important role in complex finger movement. Brain 116(Pt 6):1387-1398.

Shimizu T, Hosaki A, Hino T, Sato M, Komori T, Hirai S, Rossini PM (2002) Motor cortical disinhibition in the unaffected hemisphere after unilateral cortical stroke. Brain 125:1896-1907.

Sugiyama Y, Higo N, Yoshino-Saito K, Murata Y, Nishimura Y, Oishi $\mathrm{T}$, Isa T (2013) Effects of early versus late rehabilitative training on manual dexterity after corticospinal tract lesion in macaque monkeys. J Neurophysiol 109:2853-2865

Wannier T, Schmidlin E, Bloch J, Rouiller EM (2005) A unilateral section of the corticospinal tract at cervical level in primate does not lead to measurable cell loss in motor cortex. J Neurotrauma 22:703-717.

Wannier-Morino P, Schmidlin E, Freund P, Belhaj-Saif A, Bloch J, Mir A, Schwab ME, Rouiller EM, Wannier T (2008) Fate of rubrospinal neurons after unilateral section of the cervical spinal cord in adult macaque monkeys: effects of an antibody treatment neutralizing Nogo-A. Brain Res 1217:96-109.

Ward NS, Brown MM, Thompson AJ, Frackowiak RS (2003) Neural correlates of motor recovery after stroke: a longitudinal fMRI study. Brain 126:2476-2496.

Wyss AF, Hamadjida A, Savidan J, Liu Y, Bashir S, Mir A, Schwab ME, Rouiller EM, Belhaj-Saif A (2013) Long-term motor cortical map changes following unilateral lesion of the hand representation in the motor cortex in macaque monkeys showing functional recovery of hand functions. Restor Neurol Neurosci 31:733-760.

Xerri C, Merzenich MM, Peterson BE, Jenkins W (1998) Plasticity of primary somatosensory cortex paralleling sensorimotor skill recovery from stroke in adult monkeys. J Neurophysiol 79:2119-2148. 
Xu W, Cormier R, Fu T, Covey DF, Isenberg KE, Zorumski CF Mennerick S (2000) Slow death of postnatal hippocampal neurons by GABA(A) receptor overactivation. J Neurosci 20:3147-3156.

Yarosh CA, Hoffman DS, Strick PL (2004) Deficits in movements of the wrist ipsilateral to a stroke in hemiparetic subjects. J Neurophysiol 92:3276-3285.

\section{APPENDIX A. SUPPLEMENTARY DATA}

Supplementary data associated with this article can be found, in the online version, at http://dx.doi.org/10.1016/ j.neuroscience.2017.06.018. 